8-1988

\title{
Effect of Closed Classical Orbits on Quantum Spectra: Ionization of Atoms in a Magnetic Field. II. Derivation of Formulas
}

M. L. Du

John B. Delos

William \& Mary, jbdelos@wm.edu

Follow this and additional works at: https://scholarworks.wm.edu/aspubs

Part of the Physics Commons

\section{Recommended Citation}

Du, M. L. and Delos, John B., Effect of Closed Classical Orbits on Quantum Spectra: Ionization of Atoms in a Magnetic Field. II. Derivation of Formulas (1988). Physical Review A, 38(4), 1913-1930.

https://doi.org/10.1103/PhysRevA.38.1913

This Article is brought to you for free and open access by the Arts and Sciences at W\&M ScholarWorks. It has been accepted for inclusion in Arts \& Sciences Articles by an authorized administrator of W\&M ScholarWorks. For more information, please contact scholarworks@wm.edu. 


\title{
Effect of closed classical orbits on quantum spectra: Ionization of atoms in a magnetic field. II. Derivation of formulas
}

\author{
M. L. Du and J. B. Delos \\ Joint Institute for Laboratory Astrophysics, University of Colorado and National Bureau of Standards, \\ Boulder, Colorado 80309-0440 \\ and Department of Physics, College of William and Mary, Williamsburg, Virginia 23185*
}

(Received 30 November 1987; revised manuscript received 15 April 1988)

\begin{abstract}
A formula is derived for oscillations in the near-threshold absorption spectrum of an atom in a magnetic field. Three approximations are used. (1) Near the atomic nucleus, the diamagnetic field is negligible. (2) Far from the nucleus, the waves propagate semiclassically. (3) Returning waves are similar to (cylindrically modified) Coulomb-scattering waves. With use of these approximations, together with the physical picture described in the accompanying paper, an algorithm is specified for calculation of the spectrum.
\end{abstract}

\section{INTRODUCTION}

This is the second of two papers dealing with the effect of closed classical trajectories on quantum spectra. In the first paper, we explained the physical picture, and we stated a formula that connects closed classical orbits with oscillations in the absorption spectrum of a hydrogen atom in a magnetic field. ${ }^{1}$

The purpose of this paper is to present a derivation of that formula. The derivation is long, but it is quite straightforward, provided that the physical picture discussed in paper $I$ is kept in mind. Let us recall that physical picture. When a laser is applied to an atom in a magnetic field, the atom may absorb a photon. When it does, the electron goes into a near-zero-energy outgoing Coulomb wave. This wave then propagates away from the nucleus. Sufficiently far from the nucleus, the wave propagates according to semiclassical mechanics, and it is correlated with classical trajectories. The wave fronts are perpendicular to the trajectories, and the waves propagate along the trajectories. Eventually the trajectories and the wave fronts are turned back by the magnetic field; some of the orbits return to the vicinity of the nucleus, and the associated waves (now incoming) interfere with the outgoing waves to produce the observed oscillations in the absorption spectrum. (See Fig. 1.)

Since in this system classical trajectories are chaotic, it would be impossible to find all of the trajectories that propagate away from and return to the vicinity of the nucleus. However, since we want to calculate the absorption spectrum only to a certain resolution $\Delta E$, we include only those paths which return in a time less than $T_{\max }=2 \pi \hbar / \Delta E$.

An additional complication of the theory arises from the fact that the semiclassical approximation becomes reliable only outside the vicinity of the nucleus. We therefore use a quantum partial-wave expansion close to the nucleus and the semiclassical approximation outside of this region. The two approximations are joined on a boundary sphere of radius $r_{b} \sim 50 a_{0}$.

The paper is organized as follows. In Sec. II the photon-absorption rate is expressed by the average oscillator-strength density $\overline{D f}(E)$. This quantity is related to an energy-averaged Green's function. In Sec. III formulas are presented for propagation of waves using a semiclassical approximation. The formulas are reduced when the cylindrical symmetry of the system is considered. In Sec. IV the behavior of waves in the vicinity of the nucleus is described. The initial state and dipole operator are familiar. The outgoing wave is easy to describe. Detailed analysis is required for a description of the returning waves. In Sec. V the results of Secs. II-IV are put together to derive the formula which describes oscillations in the spectrum. In Sec. VI an algorithm for computation of the spectrum to a specified resolution is described.

There is nothing difficult in this paper, but the full analysis requires a lot of mathematical details, which we have presented as compactly as possible. The title and the first sentence of each subsection tell what happens therein. We emphasize the importance of keeping the physical picture in mind, to avoid getting lost in the formulas. Constant reference to Fig. 1 should be helpful.

\section{BASIC FORMULAS FOR SPECTRA}

Fundamental quantities needed for quantitative calculation of spectra are defined, and relationships between them are derived. The photon-absorption rate is related to an average oscillator-strength density, and the latter quantity is related to a matrix element of an energyaveraged Green's function.

\section{A. The photon-absorption rate is related to the average oscillator-strength density $\overline{D f}(E)$}

Given a collection of $N_{i}$ one-electron atoms in an initial quantum state $\psi_{i}$ of energy $E_{i}$, if a radiation field is applied to the atoms, then the rate of absorption of photons, or the rate of production of atoms in an excited state $\psi_{f}$ (energy $E_{f}$ ), is 


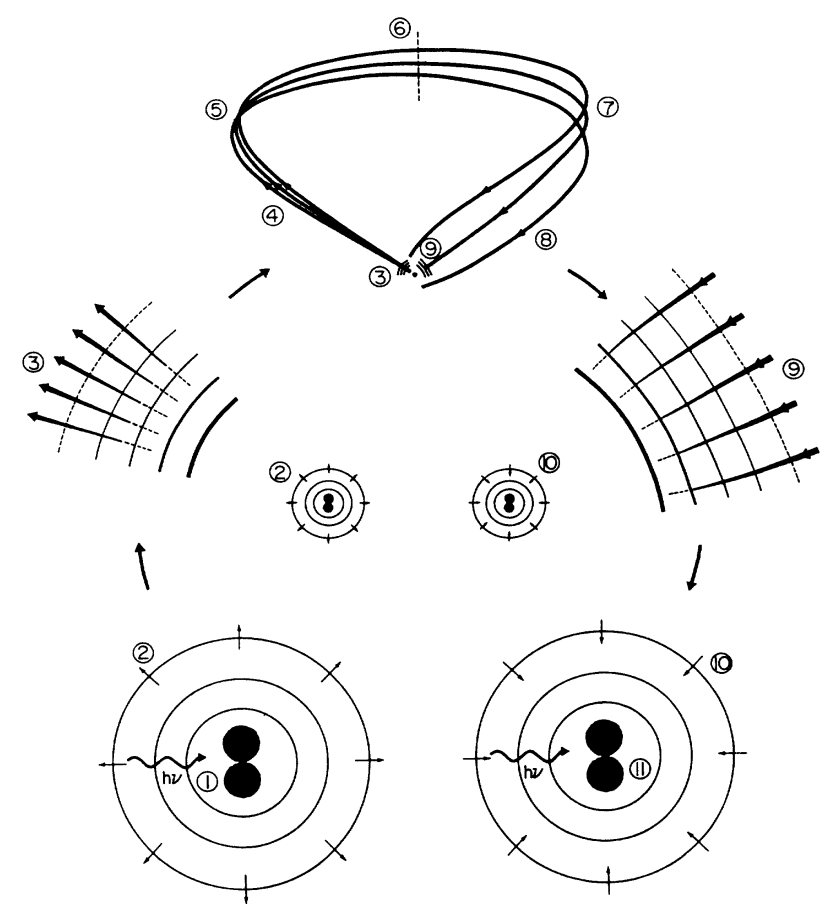

FIG. 1. Physical picture of the absorption process. (1) The atom is initially in the $2 p_{z}$ state, with the oscillating field due to the laser present. (2) The oscillating field produces zero-energy Coulomb waves, which propagate outward in all directions. (3) For distances greater than about $50 a_{0}$, a semiclassical approximation is appropriate, and we can propagate the wave outward by following classical trajectories. (4) A pencil of trajectories propagates outward, encounters a caustic (5), a focus (6), and another caustic (7). This group of trajectories started out in such a direction that it turned around and returned toward the atom (8). Around $50 a_{0}$, we describe it as an incoming zeroenergy Coulomb wave (9), which continues to propagate inward (10), until it overlaps with the initial $2 p_{z}$ state (11). Interference between steadily produced outgoing and incoming waves leads to oscillations in the absorption spectrum. (The sizes of the first and last parts of the figure are about $10 a_{0}$, the sizes of the second and fourth are about $60 a_{0}$, and the size of the third is about $3000 a_{0}$.)

$$
\frac{d N_{f}}{d t}=B_{f i} N_{i} I(\omega) .
$$

Here $I(\omega) d \omega$ is the energy flux density (energy per unit area per unit time) in the frequency range $d \omega$. (It is assumed that the range of energies in the photon beam is large compared to the natural linewidth for the transition.) $B_{f i}$ is the induced absorption coefficient between initial and final states. In many textbooks on quantum mechanics ${ }^{2}$ it is shown that $B_{f i}$ is given by

$$
B_{f i}=\frac{4 \pi^{2} e^{2}}{\hbar^{2} c}\left|\left\langle\psi_{f}|D| \psi_{i}\right\rangle\right|^{2},
$$

where $\psi_{i}$ and $\psi_{f}$ are the initial and final quantum states of the atom, $-e$ is the electron charge, $c$ is the speed of light, and $\hbar$ is the Planck constant over $2 \pi$. D is the "dipole-coordinate" operator, equal to the projection of the electron coordinate $\mathbf{r}=\widehat{\mathbf{i}} x+\widehat{\mathbf{j}} y+\hat{\mathbf{k}} z$ onto the direction of polarization of the field,

$$
D=\mathbf{r} \cdot \mathbf{A}_{0} /\left|\mathbf{A}_{0}\right|,
$$

where the vector potential for the electromagnetic wave is given $\mathrm{by}^{2}$

$$
\mathbf{A}(r, t)=\mathbf{A}^{0} \exp [i(\mathbf{k} \cdot \mathbf{r}-\omega t)]+\mathbf{A}^{0 *} \exp [-i(\mathbf{k} \cdot \mathbf{r}-\omega t)] .
$$

The operator $D$ can be written in the form

$$
\begin{aligned}
D & =a^{+}(x+i y)+a^{-}(x-i y)+a^{0} z \\
& =r\left(a^{+} \sin \theta e^{i \phi}+a^{-} \sin \theta e^{-i \phi}+a^{0} \cos \theta\right),
\end{aligned}
$$

where $a^{+}, a^{-}$, and $a^{0}$ are polarization coefficients for the radiation field,

$$
\begin{aligned}
& a^{+}=\frac{1}{2}\left(A_{x}^{0}-i A_{y}^{0}\right) /\left|\mathbf{A}^{0}\right|, \\
& a^{-}=\frac{1}{2}\left(A_{x}^{0}+i A_{y}^{0}\right) /\left|\mathbf{A}^{0}\right|, \\
& a^{0}=A_{z}^{0} /\left|\mathbf{A}^{0}\right| .
\end{aligned}
$$

For example, if the light is linearly polarized with electric field along the $z$ axis, then $a^{0}=1$ and $a^{+}=a^{-}=0$, while if the light is circularly polarized and traveling in the $z$ direction, then $a^{0}=0$, and either $a^{+}=1$ and $a^{-}=0$ (giving transitions having $\Delta m+1$ ) or $a^{+}=0$ and $a^{-}=1$ (giving transitions having $\Delta m=-1) ; m \hbar$ is the component of electronic angular momentum on the $z$ axis.

In the experiments of interest to us, the energy width of the photon beam is large compared with the spacing between the energy levels, and so transitions occur from a given initial state to many final states. As a consequence, the measured absorption rate is

$$
\frac{d N}{d t}=\int \frac{d N_{f}}{d t} \rho\left(E_{f}\right) d E_{f},
$$

where $\rho\left(E_{f}\right)$ is the density of states of the system at energy $E_{f}$. For bound states with discrete energy levels, $\rho\left(E_{f}\right)$ is a sum of $\delta$ functions peaked at each discrete energy level $E_{n}$,

$$
\rho\left(E_{f}\right)=\sum_{n} \delta\left(E_{f}-E_{n}\right)
$$

while for free states with a continuous range of levels, by enclosing the whole system in a box of finite volume $V$, one finds that $\rho\left(E_{f}\right)$ goes to infinity and $\psi_{f}$ goes to zero in such a way that $\left|\psi_{f}\right|^{2} \rho\left(E_{f}\right)$ approaches a finite limit as $V \rightarrow \infty$.

It is convenient to write the photon intensity in the form

$$
I(\omega) d \omega=I_{0} g_{\text {expt }}\left(E_{f}-E\right) d E_{f},
$$

where

$$
I_{0}=\int I(\omega) d \omega
$$

is the integrated intensity of the laser beam and 


$$
E=E_{i}+\hbar \bar{\omega}
$$

is the energy of the initial state plus the average energy $\hbar \bar{\omega}$ of the laser photons. The function $g_{\text {expt }}\left(E_{f}-E\right)$ is a convolution function representing the line shape of the laser beam-the intensity at energy $E_{f}$ when the laser is tuned so that its maximum intensity occurs at energy $E$.

The measured absorption rate is obtained by combining (2.1), (2.3), and (2.5),

$$
\frac{d N}{d t}=I_{0} N_{i} \int B_{f i} \rho\left(E_{f}\right) g_{\text {expt }}\left(E_{f}-E\right) d E_{f} .
$$

Obviously the observed average absorption spectrum depends upon the width of the convolution function, but normally it should not be sensitive to the detailed shape of this function.

In theoretical calculations, the oscillator strength is often preferred over the induced absorption coefficient. For a discrete transition, the oscillator strength is defined as

$$
\begin{aligned}
f_{f i} & \equiv \frac{2 m_{e}\left(E_{f}-E_{i}\right)}{\hbar^{2}}\left|\left\langle\psi_{f}|D| \psi_{i}\right\rangle\right|^{2} \\
& =\frac{m_{e} c\left(E_{f}-E_{i}\right)}{2 \pi^{2} e^{2}} B_{f i},
\end{aligned}
$$

where $m_{e}$ is the mass of the electron. When transitions occur to a group of unresolved final states, it is appropriate to define the oscillator-strength density (the oscillator strength per unit increment of energy) $D f\left(E_{f}\right)$ as

$$
D f\left(E_{f}\right) \equiv f_{f i} \rho\left(E_{f}\right)
$$

and to define the experimentally averaged oscillatorstrength density by the formula

$$
\begin{array}{r}
\overline{D f}_{\text {expt }}(E) \equiv\left(E-E_{i}\right) \int\left(E_{f}-E_{i}\right)^{-1} D f\left(E_{f}\right) \\
\times g_{\text {expt }}\left(E_{f}-E\right) d E_{f} .
\end{array}
$$

Normally the laser beam is narrow enough that $\left(E_{f}-E_{i}\right) \simeq\left(E-E_{i}\right)$ over the significant range of $E_{f}$, and $\overline{D f}_{\text {expt }}(E)$ is a simple average of $D f\left(E_{f}\right)$,

$$
\overline{D f}_{\text {expt }}(E) \simeq \int D f\left(E_{f}\right) g_{\text {expt }}\left(E_{f}-E\right) d E_{f}
$$

Combining (2.7a) with (2.9a) we find

$\overline{D f}_{\mathrm{expt}}(E)=\frac{m_{e} c\left(E-E_{i}\right)}{2 \pi^{2} e^{2}} \int B_{f i} \rho\left(E_{f}\right) g_{\mathrm{expt}}\left(E_{f}-E\right) d E_{f}$.

Hence the measured absorption rate (2.6) can be expressed in terms of $\overline{D f}_{\text {expt }}(E)$ as

$$
\frac{d N}{d t}=I_{0} N_{i} \frac{2 \pi^{2} e^{2}}{m_{e} c\left(E-E_{i}\right)} \overline{D f}_{\mathrm{expt}}(E) \text {. }
$$

The goal of the theory developed below is the theoretical calculation of the average oscillator-strength density.

\section{B. The propagator and Green's function are defined}

Given a time-independent quantum Hamiltonian operator $H\left(-i \hbar \nabla_{q}, \mathbf{q}\right)$, the propagator $K\left(\mathbf{q}, t ; \mathbf{q}^{\prime}, t^{\prime}\right)$ is defined as the coordinate representation of the evolution operator $^{3}$

$$
\begin{gathered}
\hat{K}\left(t, t^{\prime}\right) \equiv \exp \left[-i\left(t-t^{\prime}\right) H / \hbar\right]=\left[\hat{K}\left(t^{\prime}, t\right)\right]^{\dagger} \\
K\left(\mathbf{q}, t ; \mathbf{q}^{\prime} t^{\prime}\right)=\left\langle\mathbf{q}\left|\hat{K}\left(t, t^{\prime}\right)\right| \mathbf{q}^{\prime}\right\rangle \\
=\int \psi_{E^{\prime \prime}}(\mathbf{q}) \psi_{E^{\prime \prime}}^{*}\left(\mathbf{q}^{\prime}\right) \rho\left(E^{\prime \prime}\right) \\
\times e^{-i E^{\prime \prime}\left(t-t^{\prime}\right) / \hbar} d E^{\prime \prime}
\end{gathered}
$$

It is also convenient to define the "forward propagator" $K^{+}$, such that

$$
K^{+}\left(\mathbf{q}, t ; \mathbf{q}^{\prime} t^{\prime}\right) \equiv\left\{\begin{array}{l}
K\left(\mathbf{q}, t ; \mathbf{q}^{\prime} t^{\prime}\right) \text { for } t>t^{\prime} \\
0 \text { for } t<t^{\prime}
\end{array}\right.
$$

The outgoing-wave Green's function is

$$
\begin{aligned}
G^{+}\left(\mathbf{q}, \mathbf{q}^{\prime} ; E\right) & =\left\langle\mathbf{q}|\hat{G}+| \mathbf{q}^{\prime}\right\rangle \\
& =\left\langle\mathbf{q}\left|(E-H)^{-1}\right| \mathbf{q}^{\prime}\right\rangle \\
& =\frac{1}{i \hbar} \int_{0}^{\infty} d t K^{+}\left(\mathbf{q}, t ; \mathbf{q}^{\prime}, 0\right) \exp \left[\frac{i E t}{\hbar}\right),
\end{aligned}
$$

where $E=E+\varepsilon i$ and $\varepsilon \rightarrow+0$. All quantum dynamical properties of a system can be calculated if the propagator or Green's function is known.

\section{An energy-averaged Green's function is related to the finite-time propagator}

If the propagator is known for times $t$ up to some maximum, $0 \leq t \leq T$, then we can calculate a "finiteresolution" or "energy-averaged" Green's function. The range of energy averaging $\Delta E$ is related to the maximum time $T$ by the formula $T \Delta E \sim 2 \pi \hbar$.

Let us define

$\bar{G}_{g}\left(\mathbf{q}, \mathbf{q}^{\prime} ; E\right) \equiv(i \hbar)^{-1} \int_{0}^{\infty} K^{+}\left(\mathbf{q}, t ; \mathbf{q}^{\prime}, 0\right) \widetilde{g}(t) \exp (i E t / \hbar) d t$,

where $\widetilde{g}(t)$ is a general cutoff function. We define $\widetilde{g}(t)$ to be symmetric in time, so $\widetilde{g}(-t)=\widetilde{g}(t)$. It is easy to prove that $\bar{G}_{g}$ is an energy-averaged Green's function, specifically,

$\bar{G}_{g}\left(\mathbf{q}, \mathbf{q}^{\prime} ; E\right)=\int G^{+}\left(\mathbf{q}, \mathbf{q}^{\prime} ; E^{\prime}\right) g\left(E-E^{\prime}\right) d E^{\prime}$,

where

$$
\begin{aligned}
g(E) & =\frac{1}{2 \pi \hbar} \int_{-\infty}^{+\infty} \widetilde{g}(t) e^{i E t / \hbar} d t \\
& =\frac{1}{\pi \hbar} \int_{0}^{\infty} \widetilde{g}(t) \cos (E t / \hbar) d t .
\end{aligned}
$$

Proof. From (2.12b), the inverse Fourier transform gives 


$$
K^{+}\left(\mathbf{q}, t ; \mathbf{q}^{\prime}, 0\right)=\frac{i}{2 \pi} \int_{-\infty}^{+\infty} G^{+}\left(\mathbf{q}, \mathbf{q}^{\prime} ; E\right) e^{-i E t / \hbar} d E
$$

Substitute this formula for $K^{+}$into Eq. (2.13a), reverse the order of integration, and use the $\delta$-function formula

$$
\frac{1}{2 \pi \hbar} \int_{-\infty}^{+\infty} \exp \left[i\left(E_{f}-E\right) t / \hbar\right] d t=\delta\left(E_{f}-E\right)
$$

to arrive at $(2.13 b)$.

\section{The oscillator-strength density is expressed in terms of the propagator and the Green's function}

The oscillator-strength density is related to matrix elements of the propagator and to those of the Green's function by the formulas

$$
\begin{gathered}
D f\left(E_{f}\right)=\frac{2 m_{e}\left(E_{f}-E_{i}\right)}{\pi \hbar^{3}} \operatorname{Re} \int_{0}^{\infty}\left\langle D \psi_{i}|\hat{K}+(t, 0)| D \psi_{i}\right\rangle \\
\times e^{i E_{f} t / \hbar} d t \\
D f\left(E_{f}\right)=-\frac{2 m_{e}\left(E_{f}-E_{i}\right)}{\pi \hbar^{2}} \operatorname{Im}\left\langle D \psi_{i}|\hat{G}+| D \psi_{i}\right\rangle .
\end{gathered}
$$

Proof. First establish the relationship

$$
\begin{aligned}
\left|\left\langle\psi_{f}|D| \psi_{i}\right\rangle\right|^{2} \rho\left(E_{f}\right)=\frac{1}{2 \pi \hbar} \int_{-\infty}^{+\infty} & \left\langle D \psi_{i}|\hat{K}(t, 0)| D \psi_{i}\right\rangle \\
& \times e^{i E_{f} t / \hbar} d t
\end{aligned}
$$

To prove this, use $(2.11 \mathrm{c})$ on the right-hand side of $(2.17)$, reverse the order of integration, and use (2.15). To prove (2.16a) from (2.17), use time-reversal symmetry of the propagator, (2.11a), and the definition of $D f,(2.7)$ and (2.8). Then (2.12b) leads from (2.16a) to (2.16b).

\section{E. An energy-averaged oscillator-strength density is related to the finite-time propagator and to the energy-averaged Green's function}

If the propagator $\hat{K}(t, 0)$ is calculated for only a finitetime interval $0 \leq t \leq T$, then an averaged oscillatorstrength density is determined, and so the spectrum can be calculated to a corresponding resolution. The same "low-resolution" spectrum can be calculated from the energy-averaged Green's function.

Let us define

$$
\overline{D f}_{g}(E) \equiv\left(E-E_{i}\right) \int\left(E^{\prime}-E_{i}\right)^{-1} D f\left(E^{\prime}\right) g\left(E^{\prime}-E\right) d E^{\prime} \text {. }
$$

It is then easy to prove that

$$
\overline{D f}_{g}(E)=-\frac{2 m_{e}\left(E-E_{i}\right)}{\pi \hbar^{2}} \operatorname{Im}\left\langle D \psi_{i}\left|\bar{G}_{g}(E)\right| D \psi_{i}\right\rangle
$$

and that

$$
\begin{gathered}
\overline{D f}_{g}(E)=\frac{2 m_{e}\left(E-E_{i}\right)}{\pi \hbar^{3}} \operatorname{Re} \int_{0}^{\infty}\left\langle D \psi_{i}|\hat{K}(t, 0)| D \psi_{i}\right\rangle \\
\times \widetilde{g}(t) e^{i E t / \hbar} d t .
\end{gathered}
$$

Proof. Equation (2.19) follows trivially from (2.18), (2.16b), and (2.13b). Then Eq. (2.20) follows from (2.19) and $(2.13 a)$.

Ideally, we should take $g\left(E^{\prime}-E\right)$ in $(2.18)$ in the same form as that for the laser profile, as in Eq. (2.5a). However, while the resulting theoretical average oscillatorstrength density should depend upon the width $\Delta E$ of the convolution function, it should not be sensitive to the detailed form of this function. Therefore we consider the special case that $\widetilde{g}(t)$ corresponds to a sharp cutoff,

$$
\widetilde{g}(t)= \begin{cases}1, & |t|<T \\ 0, & |t|>T\end{cases}
$$

In this case we use only a finite-time propagator $\hat{K}(t, 0)$, for $0 \leq t \leq T$, and the resulting oscillator-strength density is averaged over energy with the convolution function

$$
g\left(E-E^{\prime}\right)=\frac{1}{\pi} \frac{\sin \left[\left(E-E^{\prime}\right) T / \hbar\right]}{\left(E-E^{\prime}\right)} .
$$

In this way, we obtain a theoretically averaged oscillator-strength density, $\overline{D f}_{\text {theor }}(E)$. This quantity will be compared to the experimentally averaged measurements $\overline{D f}$ expt $(E)$. We take the width (in energy) of the theoretical convolution function comparable to the energy width of the laser beam. Equivalently, we evaluate the propagator up to a maximum time $T$ which is comparable to $2 \pi \hbar /$ (experimental energy resolution). Henceforth we no longer distinguish between $\overline{D f}_{\text {theor }}(E)$ and $\overline{D f}_{\text {expt }}(E)$.

\section{SEMICLASSICAL PROPAGATION OF WAVES}

After the outgoing waves are produced by the laser from the initial state $\psi_{i}$, these waves propagate forward in the combined Coulomb and magnetic fields. Sufficiently far from the nucleus, this propagation can be described using the semiclassical approximation. Here we describe the semiclassical method of propagation of waves from an initial surface.

\section{A. Applicability of the semiclassical approximation is examined}

Perfectly rigorous (necessary and sufficient) conditions for the validity of the semiclassical approximation are not known, particularly for multidimensional problems. However, for motion along a line, a set of physically reasonable criteria is generally accepted. We shall show that for our system, these criteria are satisfied for motion along the $\rho$ axis if $\rho$ is not too small. The potential energy is

$$
V(\mathbf{q})=-\frac{e^{2}}{\left(\rho^{2}+z^{2}\right)^{1 / 2}}+\frac{1}{8 m_{e}}\left(\frac{e B}{c}\right)^{2} \rho^{2} .
$$




\section{The configuration-space form of the semiclassical approximation applies in the region $10 a_{0}<\rho \lesssim 1000 a_{0}$}

It is generally agreed that the configuration-space semiclassical approximation is appropriate when the rate of change of the de Broglie wavelength $\lambda=2 \pi \hbar / p$ is small,

$$
|d \lambda / d \rho|=2 \pi \hbar\left|\frac{d p / d \rho}{p^{2}}\right|=\frac{2 \pi \hbar m_{e}|d V / d \rho|}{\left[2 m_{e}(E-V)\right]^{3 / 2}} \ll 1 .
$$

We consider $E=0$ (the ionization threshold) and $B \sim 6 \mathrm{~T}$. Consider those values of $\rho$ such that the Coulomb term dominates over the diamagnetic term,

$$
\frac{e^{2}}{\rho}>\frac{1}{8 m_{e}}\left(\frac{e B}{c}\right)^{2} \rho^{2}
$$

or

$$
\rho<2 m_{e}^{1 / 3}\left(\frac{c}{B}\right)^{2 / 3} \sim 10^{3} a_{0} .
$$

With the magnetic term neglected, $V \simeq-e^{2} / \rho$, $d V / d \rho=+e^{2} / \rho^{2}$, and condition (3.2) reduces to

$$
\rho \gg\left(\pi^{2} / 2\right) a_{0} .
$$

What is happening here is the following: as $\rho$ increases, $E-V(\rho)$ becomes small and the de Broglie wave length becomes large. This seems to suggest that the semiclassical approximation should fail. However, $d V / d \rho$ decreases even more rapidly than $E-V(\rho)$; therefore the fractional change of $\lambda$ in a wavelength becomes smaller as $\rho$ increases.

\section{The momentum-space form of the semiclassical approximation is appropriate in the region $1000 a_{0}<\rho$}

At larger $\rho$, condition (3.2) is violated, as a turning point is approached $[(E-V) \rightarrow 0]$. Nevertheless, the semiclassical approximation can still give good results, because the approximation can be made in momentum space instead of in configuration space. By means of a long analysis ${ }^{4}$ (which is not presented here) one finds that a corresponding condition for validity of the momentum-space semiclassical approximation is

$$
\left|\frac{\hbar \frac{d^{3} V}{d \rho^{3}} \frac{d \rho}{d p}}{\frac{d V}{d \rho}}\right| \ll 1 .
$$

At the turning point, $d \rho / d p=0$, so the condition is certainly satisfied. As $\rho$ decreases, the left-hand side of (3.4) increases monotonically such that when $\rho \sim 1000 a_{0}$, the left-hand side turns out to be $\frac{1}{30}$. Therefore the momentum space form of the semiclassical approximation is valid at large $\rho$.

Since the configuration-space form of the semiclassical approximation is valid for $10 a_{0}<\rho<1000 a_{0}$, and the momentum-space form of it is valid for $1000 a_{0}<\rho$, we conclude that the approximation is valid everywhere ex- cept close to the nucleus. (There we use the Coulomb approximation described later.)

\section{B. Wave functions can be calculated from classical trajectories}

The semiclassical approximation provides a method for propagation of waves if the wave function is known on an initial surface. Consider a three-dimensional system with coordinates q. Consider a two-dimensional surface in this three-dimensional space, and let $q^{0}$ represent two coordinates intrinsic to the surface. Let a wave function be specified on this initial surface as

$$
\psi\left(\mathrm{q}^{0}\right)=A\left(\mathrm{q}^{0}\right) \exp \left[i S\left(\mathrm{q}^{0}\right) / \hbar\right] .
$$

A semiclassical approximation to the solution $\psi(q)$ to the Schrödinger equation is given $\mathrm{by}^{5(\mathrm{a}), 5(\mathrm{~b})}$

$$
\psi(\mathbf{q})=\sum_{k} \psi\left(\mathbf{q}_{k}^{0}\right) A_{k}(\mathbf{q}) e^{i\left[S_{k}(\mathbf{q}) / \hbar-\mu_{k} \pi / 2\right]},
$$

where

$$
\begin{aligned}
& S_{k}(\mathbf{q})=S\left(\mathbf{q}^{0}\right)+\int_{q^{0}}^{q} \mathbf{p} \cdot d \mathbf{q}, \\
& A_{k}(\mathbf{q})=\left\lfloor\frac{J\left(t=0, \mathbf{q}_{k}^{0}\right)}{J\left(t, \mathbf{q}_{k}^{0}\right)}\right]^{1 / 2}, \\
& J\left(t, \mathbf{q}_{k}^{0}\right)=\left\lfloor\operatorname{det}\left[\frac{\partial^{2} \mathbf{q}_{k}^{0}\left(t, \mathbf{q}_{k}^{0}\right)}{\partial\left(t, \mathbf{q}_{k}^{0}\right)}\right] \mid .\right.
\end{aligned}
$$

The integral (3.7) is evaluated on a classical trajectory having energy $E$, emanating from the initial surface at $\mathbf{q}_{k}^{0}$ and arriving at q. The sum in (3.6) is over all trajectories which arrive at the point $q$ from different points $q_{k}^{0}$ on the initial surface. The Jacobian (3.9) is evaluated by examining the divergence of adjacent trajectories from each central trajectory going from $\mathbf{q}_{k}^{0}$ to $\mathbf{q}$. The quantity $\mu_{k}$ in (3.6) is the Maslov index, and it will be discussed later. A full explanation of this formula is given in Refs. 5(a) and 5(b).

\section{Cylindrical symmetry permits reduction to two dimensions}

In Sec. $\mathrm{V}$ we will use the semiclassical approximation outside a sphere of radius $r_{0} \sim 30 a_{0}-50 a_{0}$ centered on the nucleus. The Hamiltonian has cylindrical symmetry, and this allows simplification of the formulas.

The $z$ component of the angular momentum $p_{\phi}=L_{z}=m \hbar$ is conserved, and the azimuthal angle is an ignorable coordinate. Initial coordinates for the sphere are the polar and azimuthal angles $\alpha=\left(\theta^{0}, \phi^{0}\right)$. If a trajectory of given energy and given $L_{z}$ is launched from the sphere, then the time development of $r(t), \theta(t)$, and $\phi(t)$ depend upon the initial polar angle $\theta^{0}$ (and of course upon the initial momenta, $p_{r}^{0} p_{\theta}^{0}$ ), but not upon the initial azimuthal angle $\phi^{0}$.

Integration of the equations of motion gives the coordinates $r, \theta$, and $\phi$ as functions of time and of the initial polar angle, 
$r=r\left(t, \theta^{0}\right)$,

$\theta=\theta\left(t, \theta^{0}\right)$,

$\phi=\phi\left(t, \theta^{0}\right)=\phi^{0}+\int \frac{m \hbar}{\left\{r\left(t, \theta^{0}\right) \sin \left[\theta\left(t, \theta^{0}\right)\right]\right\}^{2}} d t$.

The phase of the wave function is calculated from the classical action

$$
S(\mathrm{q})=S_{2}(r, \theta)+m \hbar\left(\phi-\phi_{0}\right)+S\left(r^{0}, \theta^{0}, \phi^{0}\right),
$$

where $S_{2}(r, \theta)$ is the reduced, two-dimensional action associated with the $r$ and $\theta$ motion,

$$
S_{2}(r, \theta)=\int\left[p_{r} \frac{d r}{d t}+p_{\theta} \frac{d \theta}{d t}\right] d t
$$

The integrals are always calculated on the trajectory leading from the initial sphere to the final point $(\rho, z)$.

For the amplitude factor $A(q)$ we need the Jacobian

$$
J\left(t, \theta^{0}\right)=\frac{\partial(x, y, z)}{\partial\left(t, \theta^{0}, \phi^{0}\right)}=r^{2} \sin \theta J_{2}\left(t, \theta^{0}\right),
$$

where

$$
J_{2}\left(t, \theta^{0}\right)=\left|\begin{array}{ll}
\frac{\partial r}{\partial t} & \frac{\partial \theta}{\partial t} \\
\frac{\partial r}{\partial \theta_{0}} & \frac{\partial \theta}{\partial \theta_{0}}
\end{array}\right| .
$$

Therefore the three-dimensional $A(\mathbf{q})$ is related to a two-dimensional quantity

$$
A_{2}(r, \theta) \equiv\left|\frac{J_{2}\left(0, \theta^{0}\right)}{J_{2}\left(t, \theta^{0}\right)}\right|^{1 / 2}
$$

by the formula

$$
A(\mathbf{q})=A_{2}(r, \theta)\left|\frac{r_{0}^{2} \sin \theta_{0}}{r^{2} \sin \theta}\right|^{1 / 2} .
$$

\section{The Maslov index is calculated by counting caustics and foci}

Caustics and focal points are singular points where $A$ (q) goes to infinity because $J(q)$ goes through zero. They produce additional phase shifts of the wave, and these phase shifts are described by the Maslov index. The singular points are shown for a certain group of trajectories in Fig. 1.

Caustics are envelopes or boundaries of a family of trajectories. At a caustic the trajectories curve back over each other, leaving a boundary between a classically allowed and a classically forbidden region. Near points 5 and 7 in Fig. 1 are the simplest type of caustic, known as a "fold." It has been proved that when a trajectory passes through this particular type of fold caustic, then the Maslov index increases by 1 , and the wave undergoes a phase loss of $\pi / 2$.

Near $\rho=0$ there is another type of singular region. For $m=0$, trajectories can converge onto the $z$ axis from all directions, forming a focus. In Cartesian coordinates $x(t)$ and $y(t)$ pass linearly through zero; in cylindrical coordinates $\rho(t)$ goes to zero and $d \rho / d t$ changes sign discontinuously. It is possible to prove that this type of focus also produces a phase loss of $\pi / 2$. If $m$ is not zero, the focus becomes an ordinary "fold" caustic, with the forbidden region at small $\rho$. The same phase loss of $\pi / 2$ is produced.

In our calculations and examinations of pictures of trajectories, fold caustics and foci are the only types of singular points we have found. Furthermore, in the trajectories we have examined, we have found a fold caustic close to each point at which $\rho(t)$ passes through a maximum. For $m=0$ there is a focus each time $\rho(t)$ touches zero, and for $m \neq 0$ there is a fold each time $\rho(t)$ goes through a minimum. We have not seen any other caustics.

It follows from these observations that for this system, the Maslov index for an orbit is equal to the number of maxima plus the number of minima or zeros through which $\rho(t)$ passes.

\section{E. Trajectories outgoing from a point give}

the semiclassical approximation to the Green's function

The Green's function $G^{+}\left(\mathbf{q}, \mathbf{q}^{\prime} ; E\right)$ satisfies the homogeneous stationary Schrödinger equation for all $\mathbf{q} \neq \mathbf{q}^{\prime}$. Therefore a semiclassical approximation to $G^{+}\left(\mathbf{q}, \mathbf{q}^{\prime} ; E\right)$ can be calculated by the procedure stated in Sec. III B, provided that boundary conditions are chosen correctly.

The initial surface appropriate for calculating the Green's function is a tiny sphere surrounding the point $\mathbf{q}^{\prime}$; the initial momenta are directed outward from this sphere, and on every point on the sphere $\psi\left(q^{0}\right)$ is taken to be a constant, denoted by $C\left(\mathbf{q}^{\prime}\right)$. At each $q$ through which a trajectory passes, there is a contribution to $G\left(\mathbf{q}, \mathbf{q}^{\prime} ; E\right)$ equal to

$$
C\left(\mathbf{q}^{\prime}\right) A\left(\mathbf{q} ; \mathbf{q}^{\prime}\right) \exp \left[i S\left(\mathbf{q} ; \mathbf{q}^{\prime}\right) / \hbar-i \mu \pi / 2\right],
$$

so if several trajectories of given energy pass from $q^{\prime}$ to $q$

$$
\begin{array}{rl}
G\left(\mathbf{q}, \mathbf{q}^{\prime} ; E\right)=\sum_{k} & C\left(\mathbf{q}^{\prime}\right) A_{k}\left(\mathbf{q} ; \mathbf{q}^{\prime}\right) \\
& \times \exp \left[i S_{k}\left(\mathbf{q} ; \mathbf{q}^{\prime}\right) / \hbar-i \mu_{k} \pi / 2\right] .
\end{array}
$$

This formula holds in the semiclassical approximation for all $\mathbf{q}$ outside of a sphere centered on the point $\mathbf{q}^{\prime}$ and not too close to a caustic or focus. For $\mathbf{q}$ close to $\mathbf{q}^{\prime}$ (or for $\mathbf{q}$ close to a caustic or focus), different formulas are needed.

A similar approximation holds for the propagator,

$$
\begin{aligned}
K\left(\mathbf{q}, \mathbf{q}^{\prime} ; t\right)=\sum_{k} & C^{\prime}\left(\mathbf{q}^{\prime}\right) A_{k}^{\prime}(\mathbf{q}) \\
& \times \exp \left[i S_{k}^{\prime}(\mathbf{q}) / \hbar-i \mu_{k}^{\prime} \pi / 2\right] .
\end{aligned}
$$

All the quantities in (3.14) are different from, but closely related to, the corresponding quantities in (3.13). For example, the relevant classical trajectories for the propagator are those that go from $\mathbf{q}^{\prime}$ to $\mathbf{q}$ in time $t$ (whereas for the Green's function the trajectories all have energy $E$ ), and $S^{\prime}(q)$ is the integral of the Lagrangian over time on the relevant classical trajectory. This approximation 
(3.14) holds for fixed $t$ in the limit $\hbar \rightarrow 0$.

In this paper we do not need to know the semiclassical formula for the propagator, but we do need to know that such a formula exists, and that it is consistent with the semiclassical formula for $G\left(\mathbf{q}, \mathbf{q}^{\prime} ; E\right)$. In particular, suppose we put the semiclassical approximation to $K\left(\mathbf{q}, \mathbf{q}^{\prime} ; t\right)$, Eq. (3.14), into the Fourier transform, Eq. $(2.12 b)$, and evaluate the integral using the stationaryphase method. Then (1) stationary-phase points occur at those times $t_{k}$ such that a trajectory of energy $E$ propagates from $\mathbf{q}^{\prime}$ to $\mathbf{q}$ in time $t_{k}$, and (2) each stationaryphase point contributes one of the semiclassical terms $A_{k} \exp \left[i\left(S_{k} / \hbar-i \mu_{k} \pi / 2\right)\right]$ to the formula for $G_{E}\left(\mathbf{q}, \mathbf{q}^{\prime}\right)$. These facts are established in Refs. 5(a) and 5(c).

\section{F. Finite-time trajectories give an approximation to the energy-averaged Green's function}

Now we state the central result, on which much of our theory is based. We showed in Sec. II C that an energyaveraged Green's function $\bar{G}+\left(\mathbf{q}, \mathbf{q}^{\prime} ; E\right)$ is related to a finite-time propagator $K\left(\mathrm{q}, t ; \mathrm{q}^{\prime}, 0\right)$ for $0<t<T$. [See Eqs. (2.13).] According to (2.21), a longer $T$ gives a higher-resolution Green's function, and shorter $T$ gives a lower-resolution Green's function. We also stated above that a semiclassical approximation to the propagator is obtained by considering trajectories that propagate from $q^{\prime}$ to $q$ in time $t$, and that when the stationary-phase method is applied to the Fourier transform, it selects trajectories of energy $E$, and gives the semiclassical formula (3.13) for $\boldsymbol{G}\left(\mathbf{q}, \mathbf{q}^{\prime} ; E\right)$.

Now let the same stationary-phase argument be applied to the energy-averaged Green's function (Fig. 2). Since it is obtained by cutting off the propagator at a finite time $T$, only stationary-phase points with $t_{k}<T$ are included. Hence we arrive again, by a different argument than that given in paper $I$, at the following important proposition.

A semiclassical approximation to the energy-averaged Green's function is obtained by including in Eq. (3.13) only those trajectories of energy $E$ which propagate from $\mathbf{q}^{\prime}$ to $\mathbf{q}$ in a time less than a fixed maximum, $T$.

\section{WAVE FUNCTIONS CLOSE TO THE NUCLEUS}

Close to the nucleus the magnetic field can be neglected, and the wave functions are those associated with a pure Coulomb field. We justify this approximation, then discuss the initial state $\psi_{i}$ and the dipole operator times the initial state $D \psi_{i}$, then the outgoing near-zero-energy Coulomb wave, and finally the returning wave.

\section{A. The diamagnetic term is negligible}

If the magnetic field is $6 \mathrm{~T}$, and if the electron stays within 100 bohr of the nucleus, then the ratio of the magnetic term to the Coulomb term in the Hamiltonian would be less than $10^{-3}$. Therefore neglecting the diamagnetic term close to the nucleus is well justified.

\section{B. Initial wave functions and dipole operator are specified}

After dropping the diamagnetic term from the Hamiltonian, what remains is the hydrogenic Hamiltonian. For the eigenfunctions,

$$
\psi_{i}=\psi_{n l m}(r, \theta, \phi)=R_{n l}(r) Y_{l m}(\theta, \phi)
$$

we use the definitions and phase conventions given by Bethe and Salpeter. ${ }^{6}$

When the dipole operator acts on these states, it connects $\Psi_{n l m}$ to $\Psi_{n, l \pm 1, m \pm 1}$,

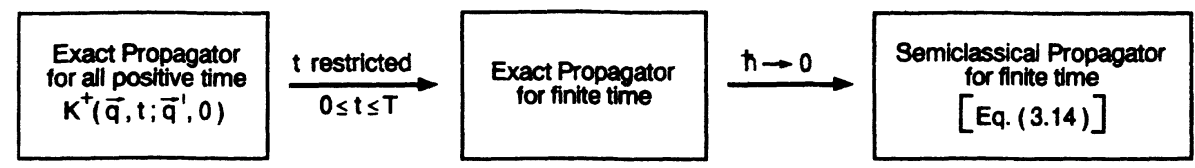

Fourier Transformation

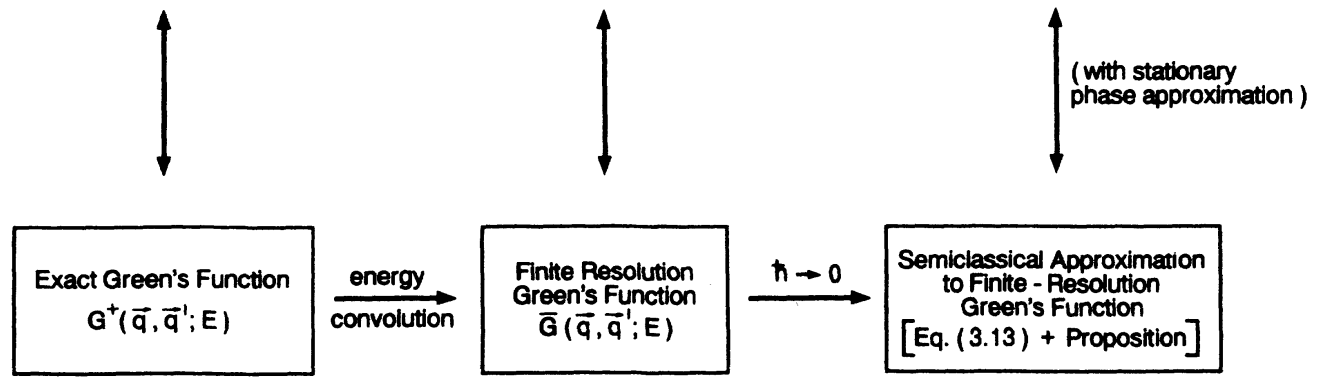

FIG. 2. Conceptual structure. In paper I we went from the exact Green's function to the semiclassical Green's function to the finite-resolution semiclassical Green's function. The somewhat stronger argument in this paper proceeds from the exact propagator to the finite-time propagator to the semiclassical finite-time propagator, and then to the finite-resolution semiclassical Green's function. 


$$
\begin{aligned}
D \psi_{i}=r R_{n l}(r)\left\{a^{+}\left[\left(\frac{(l+m+1)(l+m+2)}{(2 l+1)(2 l+3)}\right]^{1 / 2} Y_{l+1, m+1}-\left[\frac{(l-m)(l-m-1)}{(2 l+1)(2 l-1)}\right]^{1 / 2} Y_{l-1, m+1}\right]\right. \\
+a^{-}\left[-\left[\frac{(l-m+1)(l-m+2)}{(2 l+1)(2 l+3)}\right]^{1 / 2} Y_{l+1, m-1}+\left[\frac{(l+m)(l+m-1)}{(2 l+1)(2 l-1)}\right]_{l-1, m-1}^{1 / 2} Y_{l-1}\right. \\
+a^{0}\left[\left[\frac{(l+m+1)(l-m+1)}{(2 l+1)(2 l+3)}\right]^{1 / 2} Y_{l+1, m}+\left[\frac{(l+m)(l-m)}{(2 l+1)(2 l-1)}\right]_{l-1, m}^{1 / 2} Y_{l}\right.
\end{aligned}
$$

For convenience, let $b_{l^{\prime} m}^{i}$ denote the coefficients in this expression, and write $D \psi_{i}$ as

$$
D \psi_{i}=\left(\sum_{l^{\prime}, m} b_{l^{\prime} m}^{i} Y_{l^{\prime} m}\right) r R_{n l}(r) .
$$

The coefficients $b_{l^{\prime} m}^{i}$ involve the coefficients $a^{ \pm}$and $a^{0}$ describing the polarization of the radiation field and the Clebsch-Gordan coefficients in Eq. (4.2).

\section{Hydrogenic wave functions near the ionization threshold}

\section{Zero-energy radial wave functions are Bessel functions}

In general, the radial wave functions $R_{n l}(r)$ can be written in terms of confluent hypergeometric functions. However, the solution near the ionization threshold is much simpler. They are expressed in terms of Bessel functions by the formula

$$
\begin{aligned}
& R_{l}^{0, \text { reg }}(r)=J_{2 l+1}\left((8 r)^{1 / 2}\right) /(8 r)^{1 / 2} \\
& R_{l}^{0, \text { out }}(r)=H_{2 l+1}^{(1)}\left((8 r)^{1 / 2}\right) /(8 r)^{1 / 2}
\end{aligned}
$$

(the superscript zero means zero energy).

Proof. Write down the differential equation satisfied by $R_{l}^{0}(r)$ (the zero-energy radial Schrödinger equation with an attractive Coulomb field), change variables to $x=(8 r)^{1 / 2}$, and substitute $R_{l}^{0}(r)=B_{l}(x) / x$ and derive the differential equation governing $B_{l}(x)$. It is Bessel's equation of order $(2 l+1)$.

Later we will need the asymptotic approximation for the Hankel function. That formula is ${ }^{7}$

$H_{v}^{(1)}(z) \sim[2 /(\pi z)]^{1 / 2} \exp \left[i\left(z-\frac{1}{2} v \pi-\frac{1}{4} \pi\right)\right]$ as $z \rightarrow \infty$.

Accuracy for $E \neq 0$ is estimated. Equations (4.5) are exact at $E=0$. We will use these formulas as approximations also for other $E$ near zero. For energies not too different from zero, the error made by replacing the exact wave function at energy $E$ with the wave function at zero energy is small.

Our formulas will involve a dipole matrix element between the regular Bessel function and the initial radial function. Therefore we are concerned about the difference between the exact regular wave function $R_{l}^{E}(r)$ and the zero-energy regular wave function $R_{l}^{0}(r)$ for $r \lesssim r_{0} \sim n^{2}=4$. A simple estimate of this difference is obtained by using the Wentzel-Kramers-Brillouin (WKB) approximation for the zero-energy wave function $R_{l}^{0}(r)$ and for the wave function at some other energy $E, R_{l}^{E}(r)$. The difference between the phases of these two wave functions is

$$
\begin{aligned}
\Delta(\text { phase }) & =\int_{0}^{r_{0}}\left(p_{r}^{E}-p_{r}^{0}\right) d r \\
& =\int_{0}^{r_{0}}\left[\left(2 E+\frac{2}{r}\right]^{1 / 2}-\left(\frac{2}{r}\right)^{1 / 2}\right] d r .
\end{aligned}
$$

If $r_{0}$ is taken as $5 a_{0}$, then expanding the integrand in powers of energy $E$, the phase difference is estimated as

$$
\Delta(\text { phase }) \sim \frac{\sqrt{2}}{3} r_{0}^{3 / 2} E \sim 5.27 E \text {. }
$$

For $|E| \leq 100 \mathrm{~cm}^{-1}$, for example, the phase difference would be smaller than $2.4 \times 10^{-3}$. Therefore the relative error in the matrix elements should be no more than a few tenths of a percent.

\section{The Coulomb Green's function $G_{c}^{+}\left(q, q^{\prime}\right)$ near the ionization threshold is specified}

To obtain the Green's function $G^{+}\left(\mathbf{q}, \mathbf{q}^{\prime} ; E\right)$ we need the corresponding Green's function for a Coulomb field. The expansion of the Green's function in spherical harmonics is

$$
G_{c}^{+}\left(\mathbf{q}, \mathbf{q}^{\prime} ; E\right)=\sum_{l, m} Y_{l m}^{*}\left(\theta^{\prime}, \phi^{\prime}\right) g_{l}^{E}\left(r, r^{\prime}\right) Y_{l m}(\theta, \phi),
$$

with the radial part of the Green's function $g_{l}^{E}\left(r, r^{\prime}\right)$ given by the formula

$$
g_{l}^{E}\left(r, r^{\prime}\right)=\frac{2 R_{l}^{E, \text { reg }}\left(r_{<}\right) R_{l}^{E, \text { out }}\left(r_{>}\right)}{\left(r^{\prime}\right)^{2} W\left(R_{l}^{E, \text { reg }}\left(r^{\prime}\right), R_{l}^{E, \text { out }}\left(r^{\prime}\right)\right)},
$$

where $R_{l}^{E}$ are Coulomb functions at energy $E, R_{l}^{E \text {, reg is }}$

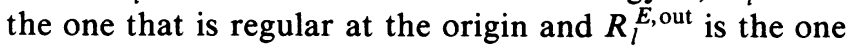
that is outgoing at large distances, $W$ is the Wronskian $W\left(R_{1}, R_{2}\right)=R_{1} R_{2}^{\prime}-R_{1}^{\prime} R_{2}, \quad r_{>}=\max \left\{r, r^{\prime}\right\}, \quad$ and $r_{<}$ $=\min \left\{r, r^{\prime}\right\}$.

For the special case $E=0$, from Eqs. (4.4) and (4.5) we find

$g_{l}^{0}\left(r, r^{\prime}\right)=(-2 \pi i) \frac{J_{2 l+1}\left[\left(8 r_{<}\right)^{1 / 2}\right] H_{2 l+1}^{(1)}\left[\left(8 r_{>}\right)^{1 / 2}\right]}{\left(r_{<} r_{>}\right)^{1 / 2}}$.

As explained below Eq. (4.6), this formula can be used in 
a range of energies close to $E=0$. Proof of formulas similar to (4.10) can be found in Ref. 8 .

\section{A radial overlap integral is defined}

The integrals $\left\langle D \psi_{i}\left|G^{+}\right| D \psi_{i}\right\rangle$ involve the overlap of the radial wave function of the initial state $R_{n l}(r)$ with the zero-energy radial wave function

$$
J_{2 l+1}\left[(8 r)^{1 / 2}\right] /(8 r)^{1 / 2} .
$$

We define

$I\left(n, l, l^{\prime}\right) \equiv \int_{0}^{\infty} R_{n l}(r) r^{3} \frac{J_{2 l^{\prime}+1}\left((8 r)^{1 / 2}\right)}{r^{1 / 2}} d r$.

As is shown in Appendix A, $I\left(n, l, l^{\prime}\right)$ can be expressed as an analytic function of $n, l$, and $l^{\prime}$. In Table I, the first few $I\left(n, l, l^{\prime}\right)$ are listed. The only relevant integrals $I\left(n, l, l^{\prime}\right)$ are those for which $l^{\prime}=l \pm 1$.

\section{The returning waves scatter from the Coulomb field}

As stated earlier, when the laser acts upon the electron in initial state $\psi_{i}$, it excites it to a near-zero-energy outgoing Coulomb wave. These outgoing waves travel in space, and some of them are turned back by the magnetic field, so they return to the vicinity of the nucleus. Here we describe these returning waves. We make the approximation that these returning waves are similar to the waves that would be obtained from a plane-wave source at infinite distance in a pure Coulomb field. This approximation will be justified numerically later (it turns out to be accurate to a few percent).

A rather complete description of Coulomb scattering is given by Schiff. ${ }^{2}$ In the conventional description, the incident direction of the waves is the $+z$ direction. Our returning waves can come from any direction, specified by a polar angle $\theta_{f}$. However, these returning waves must have a cylindrical symmetry about the $z$ axis: they depend on the azimuthal angle as $e^{i m \phi}$.

To construct an appropriate approximation to the returning waves, three steps are necessary (Fig. 3). (1) The zero-energy limit of the usual Coulomb-scattering wave function must be found. (2) That wave function must be rotated so that the electrons approach from direction $\theta_{f}$.

TABLE I. Radial overlap integrals $I(n, l, l \pm 1)$.

\begin{tabular}{llrr}
\hline \hline$n$ & $l$ & $I(n, l, l+1)$ & $I(n, l, l-1)$ \\
\hline 1 & 0 & 1.5311 & \\
2 & 0 & 4.6888 & \\
2 & 1 & 5.4142 & 1.3535 \\
3 & 0 & 9.1804 & \\
3 & 1 & 11.1283 & -1.2365 \\
3 & 2 & 9.9535 & 1.6589 \\
4 & 0 & 16.8411 & \\
4 & 1 & 17.9633 & 7.1497 \\
4 & 2 & 18.5393 & 4.0555 \\
4 & 3 & 14.0144 & 1.7518 \\
\hline \hline
\end{tabular}

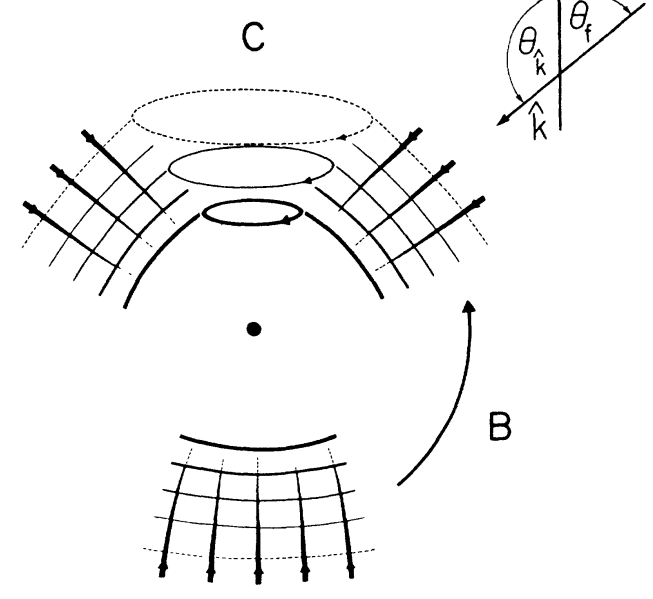

A

FIG. 3. Approximation for the returning wave is constructed by the following process. $A$ : A zero-energy Coulombscattering wave $\psi_{c}$ is calculated. The classical trajectories associated with such a wave are parabolas with focus at the nucleus; the wave fronts are also parabolic. $B$ : This wave function is rotated so that it approaches from direction $\theta_{f}$. The resulting wave function is denoted $\psi_{c, \theta_{f} \phi_{f}}$. C: This wave function is spun about the $z$ axis. We construct a cylindrically modified zeroenergy Coulomb-scattering wave $\psi_{c, \theta_{f}}^{m}$ by coherent superposition of waves $\psi_{c, \theta_{f} \phi_{f}}$ together with the factor $\exp (\operatorname{im} \phi)$. We make the approximation that the returning waves [(9) in Fig. 1] are nearly equal to a constant times $\psi_{c, \theta_{f}}^{m}$.

(3) The resulting wave function must be spun about the $z$ axis, so that electrons approach from all azimuthal angles $\phi$ and the wave function is proportional to $\exp (\operatorname{im} \phi)$. These steps are carried out below.

\section{The conventional Coulomb scattering wave function is simplified at zero energy}

We imagine a proton located at the origin of the coordinate system, and an electron at infinite distance approaching along the negative $z$ axis with asymptotic velocity $v$. The exact solution of the wave equation, including the incoming wave and the scattered wave, can be written ${ }^{2}$ as

$$
\begin{aligned}
\psi=e^{i k z} F(-i \hat{n}, 1, i k \xi) \\
=\frac{1}{\Gamma(1+i \hat{n})} \sum_{l} d_{l}(2 i k r)^{l} e^{i k r} \\
\\
\quad \times F(l+1+i \hat{n}, 2 l+2,-2 i k r) \\
\quad \times P_{l}(\cos \theta) .
\end{aligned}
$$

In (4.13),

$$
d_{l}=\frac{\Gamma(l+1+i \hat{n})}{(2 l) !}, \quad \hat{n}=-\frac{m_{e} e^{2}}{\hbar^{2} k}=-\frac{e^{2}}{\hbar v} .
$$


Here $\xi$ is the parabolic coordinate, $\xi=r-z$, and $F$ is the confluent hypergeometric function.

To evaluate this function in the zero-energy limit, we use the "well-known" expansion for the confluent hypergeometric function in terms of Bessel functions, ${ }^{7}$

$$
\begin{aligned}
\frac{F(a, b, z)}{\Gamma(b)}=e^{h z} \sum_{n=0}^{\infty} & C_{n} z^{n}(-a z)^{(1 / 2)(1-b-n)} \\
& \times J_{b-1+n}\left(2(-a z)^{1 / 2}\right),
\end{aligned}
$$

where

$$
\begin{aligned}
C_{0}=1, \quad C_{1}= & -b h, \quad C_{2}=-\frac{1}{2}(2 h-1) a+\frac{1}{2} b(b+1) h^{2}, \\
(n+1) C_{n+1}= & {[(1-2 h) n-b h] C_{n} } \\
& +[(1-2 h) a-h(h-1)(b+n-1)] C_{n-1} \\
& -h(h-1) a C_{n-2}
\end{aligned}
$$

( $h$ any real number).

Using this expansion in (4.13), it is straightforward to prove that as $v \rightarrow 0$ the wave function $\psi$ becomes

$$
\begin{aligned}
\psi \rightarrow \psi_{c} & =J_{0}(2 \sqrt{\xi}) \\
& =\sum_{l} \frac{2 l+1}{\sqrt{2}} P_{l}(\cos \theta) \frac{J_{2 l+1}\left((8 r)^{1 / 2}\right)}{r^{1 / 2}} .
\end{aligned}
$$

(Not surprisingly, this partial-wave expansion involves the regular zero-energy radial function $J_{2 l+1}\left[(8 r)^{1 / 2}\right] / \sqrt{r}$ found earlier.)

\section{The wave function is rotated to an arbitrary initial direction}

Section IV D 1 describes the zero-energy Coulomb wave function if the electron comes from the negative $z$ direction. We need the solution if the electron comes from any arbitrary direction. This solution is obtained by rotation of (4.16) (see Fig. 3).

Let $\hat{\mathbf{k}}$ be the unit vector representing the direction of motion of the incoming electron long before the collision. The angles $\left(\theta_{f}, \phi_{f}\right)$ tell the direction from which the electrons come, and the angles

$$
\theta_{k}=\pi-\theta_{f}, \quad \phi_{k}=\pi+\phi_{f}
$$

tell the initial direction of motion of the electrons.

Equations (4.16) still describe this rotated Coulomb wave if the variables $\xi$ and $\theta$ are reinterpreted. $\xi=r-\hat{\mathbf{k}} \cdot \mathbf{r}$ becomes a rotated parabolic coordinate and $\theta$ becomes the angle between $\hat{\mathbf{k}}$ and the electron position vector $\mathbf{r}$, so that $\cos \theta=\hat{\mathbf{k}} \cdot \mathbf{r} / r$. Equations (4.16) now be- come

$$
\begin{aligned}
\psi_{c, \theta_{f} \phi_{f}} & \equiv \text { rotated } \psi_{c} \\
& =J_{0}\left(2(r-\hat{\mathbf{k}} \cdot \mathbf{r})^{1 / 2}\right) \\
& =\sum_{l} \frac{2 l+1}{\sqrt{2}} P_{l}(\hat{\mathbf{k}} \cdot \mathbf{r} / r) \frac{J_{2 l+1}\left((8 r)^{1 / 2}\right)}{r^{1 / 2}} .
\end{aligned}
$$

To write Eq. (4.18) explicitly in terms of the spherical polar coordinate of $\mathbf{r}$, the following relation ${ }^{9}$ is used. Let two vectors have directions defined by polar angles $\theta_{1}, \phi_{1}$ and $\theta_{2}, \phi_{2}$, and let $\gamma$ be the angle between the two vectors; then

$$
P_{l}(\cos \gamma)=\frac{4 \pi}{2 l+1} \sum_{m} Y_{l m}^{*}\left(\theta_{1}, \phi_{1}\right) Y_{l m}\left(\theta_{2}, \phi_{2}\right) .
$$

(This relationship is independent of the phase conventions for $Y_{l m}$ 's.)

Using Eq. (4.19), if $\theta_{k} \phi_{k}$ denote the spherical polar angles of $\mathbf{k}$, and $\theta, \phi, r$ denote the spherical coordinate of $\mathbf{r}$, then the function in Eqs. (4.18) can be written as

$$
\begin{aligned}
\psi_{c, \theta_{f} \phi_{f}} & =J_{0}\left(2 \left\{r \left[1-\cos \theta_{k} \cos \theta\right.\right.\right. \\
& \left.\left.\left.-\sin \theta_{k} \sin \theta \cos \left(\phi-\phi_{k}\right)\right]\right\}^{1 / 2}\right) \\
& =\sum_{l, m} \frac{4 \pi}{\sqrt{2}} Y_{l m}^{*}\left(\theta_{k}, \phi_{k}\right) Y_{l m}(\theta, \phi) \frac{J_{2 l+1}\left[(8 r)^{1 / 2}\right]}{r^{1 / 2}} .
\end{aligned}
$$

\section{Cylindrical symmetry is regained}

The rotated wave, Eq. (4.19), is no longer cylindrically symmetric about the $z$ axis. The actual returning waves must be proportional to $e^{i m \phi}$.

To get a classical picture, imagine what happens if the two-dimensional family of trajectories in Fig. 3 is rotated about the $z$ axis to produce a three-dimensional family. Then at moderate distances from the nucleus $\left(r \sim 50 a_{0}\right)$, electrons approach the nucleus from directions specified by a fixed polar angle $\theta_{f}$, not just in the plane of the figure, but from all azimuthal angles $\phi_{f}\left(0 \leq \phi_{f}<2 \pi\right)$. What is the wave function corresponding to this situation?

Equation (4.19) describes a zero-energy Coulomb wave coming from the direction $\left(\theta_{f}, \phi_{f}\right)$. The total wave for electrons coming from polar angle $\theta_{f}$ with any azimuthal angle is a superposition of waves coming from each $\phi_{f}$.

For the case $m=0$, this cylindrical wave is

$\psi_{c, \theta_{f}}^{0} \equiv \frac{1}{2 \pi} \int_{0}^{2 \pi} \psi_{c, \theta_{f} \phi_{f}} d \phi_{k}=\frac{1}{2 \pi} \int_{0}^{2 \pi} d \phi_{k} J_{0}\left(2\left\{r\left[1-\cos \theta_{k} \cos \theta-\sin \theta_{k} \sin \theta \cos \left(\phi-\phi_{k}\right)\right]\right\}^{1 / 2}\right)$,

which is also equal to [from $(4.20 \mathrm{~b})]$

$$
\psi_{c, \theta_{f}}^{0}=\frac{1}{2 \pi} \int_{0}^{2 \pi} d \phi_{k} \sum_{l, m} \frac{4 \pi}{\sqrt{2}} Y_{l m}^{*}\left(\theta_{k}, \phi_{k}\right) Y_{l m}(\theta, \phi) \frac{J_{2 l+1}\left((8 r)^{1 / 2}\right)}{r^{1 / 2}}
$$

For $m \neq 0$, the waves have to be superposed coherently with the factor $e^{i m \phi_{k}}$. The resulting cylindrically modified 
zero-energy Coulomb wave, denoted $\psi_{c, \theta_{f}}^{m}(\mathbf{q})$, is [from Eq. (4.20a)]

$$
\psi_{c, \theta_{f}}^{m} \equiv \frac{1}{2 \pi} \int e^{i m \phi_{k}} \psi_{c, \theta_{f} \phi_{f}} d \phi_{k}=\frac{1}{2 \pi} \int_{0}^{2 \pi} d \phi_{k} e^{i m \phi_{k}} J_{0}\left(2\left\{r\left[1-\cos \theta_{k} \cos \theta-\sin \theta_{k} \sin \theta \cos \left(\phi-\phi_{k}\right)\right]\right\}^{1 / 2}\right)
$$

or [from Eq. (4.20b)]

$$
\begin{aligned}
\psi_{c, \theta_{f}}^{m} & =\frac{1}{2 \pi} \int_{0}^{2 \pi} d \phi_{k} e^{i m \phi_{k}} \sum_{l, m^{\prime}} \frac{4 \pi}{\sqrt{2}} Y_{l m^{\prime}}^{*}\left(\theta_{k}, \phi_{k}\right) Y_{l m^{\prime}}(\theta, \phi) \frac{J_{2 l+1}\left((8 r)^{1 / 2}\right)}{r^{1 / 2}} \\
& =\frac{4 \pi}{\sqrt{2}} \sum_{l \geq|m|} Y_{l m}^{*}\left(\theta_{k}, 0\right) Y_{l m}(\theta, \phi) \frac{J_{2 l+1}\left((8 r)^{1 / 2}\right)}{r^{1 / 2}} \\
& =\frac{4 \pi}{\sqrt{2}} \sum_{l \geq|m|}(-1)^{l-m} Y_{l m}^{*}\left(\theta_{f}, 0\right) Y_{l m}(\theta, \phi) \frac{J_{2 l+1}\left((8 r)^{1 / 2}\right)}{r^{1 / 2}} .
\end{aligned}
$$

\section{Asymptotic approximations give a closed form and a partial-wave expansion}

We need a closed-form expression and a partial-wave expansion for the incoming part of the cylindrically modified zero-energy Coulomb wave. The required expressions are

$$
\begin{aligned}
& \left(\psi_{c, \theta_{f}}^{m}\right)_{\mathrm{inc}}=(-1)^{m} e^{i \pi / 2} \frac{1}{2^{3 / 2} \pi} \frac{1}{\left(\sin \theta_{f}\right)^{1 / 2}} \frac{e^{-i 2\left\{r\left[1+\cos \left(\theta-\theta_{f}\right)\right]\right\}^{1 / 2}}}{(r \sin \theta)^{1 / 2}} e^{i m \phi}, \\
& \left(\psi_{c, \theta_{f}}^{m}\right)_{\mathrm{inc}}=\frac{4 \pi}{\sqrt{2}} \sum_{l \geq|m|}(-1)^{l-m} \frac{1}{2^{5 / 4} \pi^{1 / 2}} Y_{l m}^{*}\left(\theta_{f}, 0\right) Y_{l m}(\theta, \phi) \frac{\exp \left\{i\left[-(8 r)^{1 / 2}+l \pi+\frac{3}{4} \pi\right]\right\}}{r^{3 / 4}} .
\end{aligned}
$$

$\left(\psi_{c, \theta_{f}}^{m}\right)_{\text {inc }}$ means the incoming part of $\psi_{c, \theta_{f}}^{m}$. These expressions hold for $r \gtrsim 10 a_{0}$. The first holds for $\theta$ close to $\theta_{f}$ and the second is the corresponding partial-wave expansion.

Proof of Eq. (4.23a). Use the asymptotic approximation for the Bessel function

$$
J_{v}(z)=\left(\frac{2}{\pi z}\right)^{1 / 2} \cos \left(z-\frac{1}{2} v \pi-\frac{\pi}{4}\right)
$$

to obtain for the incoming part of $\psi_{c, \theta_{f}}^{m}$

$$
\begin{aligned}
\left(\psi_{c, \theta_{f}}^{m}\right)_{\text {inc }}=\frac{1}{2 \pi} \int_{0}^{2 \pi} d \phi_{k} e^{i m \phi_{k}} \frac{1}{2}\left[\frac{2}{\pi}\right]^{1 / 2} \frac{1}{\left(2\left\{r\left[1-\cos \theta_{k} \cos \theta-\sin \theta_{k} \sin \theta \cos \left(\phi_{k}-\phi\right)\right]\right\}^{1 / 2}\right)^{1 / 2}} \\
\times \exp \left(-i 2\left\{r\left[1-\cos \theta_{k} \cos \theta-\sin \theta_{k} \sin \theta \cos \left(\phi_{k}-\phi\right)\right]\right\}^{1 / 2}+\frac{\pi}{4}\right) .
\end{aligned}
$$

There are two stationary phase points for this integral, namely,

$$
\phi_{k}-\phi=0, \phi_{k}-\phi=\pi,
$$

but for the incoming wave, only the second contributes. [Recall Eqs. (4.17); $\hat{\mathbf{k}}$ is opposite to the direction of approach of the electrons.]

Using the stationary-phase approximation,

$$
\int e^{i \alpha(\phi-\hat{\phi})^{2}} f(\phi) d \phi=\left(\frac{2 \pi}{|\alpha|}\right)^{1 / 2} f(\hat{\phi}) e^{i(1 / 4) \pi \operatorname{sgn} \alpha},
$$

the result is

$$
\begin{aligned}
\left(\psi_{c, \theta_{f}}^{m}\right)_{\mathrm{inc}}= & (-1)^{m} e^{i(1 / 2) \pi} \frac{1}{2^{3 / 2} \pi} \frac{1}{\left(\sin \theta_{k}\right)^{1 / 2}} \\
& \times \frac{e^{-i 2\left\{r\left[1-\cos \left(\theta+\theta_{k}\right)\right]\right\}^{1 / 2}}}{(r \sin \theta)^{1 / 2}} e^{i m \phi},
\end{aligned}
$$

and when it is rewritten with $\pi-\theta_{f}$ replacing $\theta_{k}$, Eq (4.23a) is obtained.

Equation $(4.23 \mathrm{~b})$ is proved by putting the asymptotic formula for the Bessel function (4.24) into Eq. (4.22c) and examining the incoming part of the result.

Equations (4.23a) and (4.23b) are the required formulas for the incoming part of the cylindrically modified zeroenergy Coulomb-scattering waves, $\left[\psi_{c, \theta_{f}}^{m}(r, \theta, \phi)\right]_{\text {inc. }}$. The first is a closed-form expression and the second is a partial-wave expansion. Later we will compute the returning waves by a semiclassical approximation that is valid for $r \gtrsim 50 a_{0}$. We will make the approximation that in the region $r \sim 50 a_{0}$ and $\theta$ close to $\theta_{f}$, these returning waves are nearly equal to a constant times $\left(\psi_{c, \theta_{f}}^{m}\right)_{\text {inc }}$, using the closed-form expression (4.23a). It must follow that the returning waves have partial-wave expansion (4.23b) (times the same constant). These waves continue 
to propagate inward toward the nucleus, where Eq. $(4.22 \mathrm{c})$ provides the appropriate expression.

\section{FORMULAS FOR THE ABSORPTION SPECTRUM}

The physical picture described in paper I and in the Introduction to this paper is well expressed in the formula (2.16b) (see also Fig. 1). The initial atomic state $\left|\psi_{i}\right\rangle$ is modified by the dipole operator associated with the laser field to give $\left|D \psi_{i}\right\rangle$. The operator $G^{+}$propagates these waves outward at fixed energy. At small distances from the nucleus, $G^{+}\left|D \psi_{i}\right\rangle$ is described by a quantum partial-wave expansion (Sec. IV C). Around $50 a_{0}$ the partial-wave expansion is joined into a semiclassical wave, which propagates along trajectories, as described in Sec. III. Some of the trajectories, and their associated waves, eventually turn back toward the nucleus. Around $50 a_{0}$ the returning semiclassical waves must be joined to a quantum partial-wave expansion. This joining is accomplished using the approximation that the returning waves are similar to cylindrically modified Coulomb waves described in Sec. IV D. Finally, the incoming waves overlap with $\left\langle D \psi_{i}\right|$, giving an interference pattern in the absorption spectrum. In this section we combine all the formulas derived earlier to derive an expression for the absorption spectrum.

\section{A. The Green's function is separated into "direct" and "returning" contributions and the direct part is specified}

The Green's function $G^{+}\left(\mathbf{q}, \mathbf{q}^{\prime} ; E\right)$ represents the waves at $\mathbf{q}$ which arise from a source at $\mathbf{q}^{\prime}$. Since $G^{+}$sits between $\left\langle D \psi_{i}\right|$ and $\left|D \psi_{i}\right\rangle$, the relevant source points $\mathbf{q}^{\prime}$ all lie within a few bohrs of the nucleus. Two types of waves arrive at q. First, there are waves which propagate directly from $q^{\prime}$ to $q$ without ever leaving the vicinity of the nucleus. Second, there are waves that propagate outward from $\mathbf{q}^{\prime}$, travel to the ends of the classically allowed region, are turned back by the magnetic field, return to the vicinity of the nucleus, and finally arrive at $q^{\prime}$. The distinction between the two types is unambiguous. Therefore we can write

$$
G^{+}\left(\mathbf{q}, \mathbf{q}^{\prime} ; E\right)=G_{\mathrm{dir}}^{+}\left(\mathbf{q}, \mathbf{q}^{\prime} ; E\right)+G_{\text {ret }}^{+}\left(\mathbf{q}, \mathbf{q}^{\prime} ; E\right) .
$$

To evaluate the direct part, we notice that the diamagnetic term is negligible close to the nucleus. Therefore the direct term is just the ordinary Coulomb Green's function $G_{c}^{+}\left(\mathbf{q}, \mathbf{q}^{\prime} ; E\right)$ which was given in Eqs. (4.9)-(4.11).
From this result and the formula

$$
D f(E)=-\frac{2 m_{e}\left(E-E_{i}\right)}{\pi \hbar^{2}} \operatorname{Im}\left\langle D \psi_{i}\left|\hat{G}_{\mathrm{dir}}^{+}+\hat{G}_{\mathrm{ret}}^{+}\right| D \psi_{i}\right\rangle,
$$

it follows that the direct contribution to $G^{+}$gives the absorption spectrum that would occur in the absence of a magnetic field.

\section{B. The returning part is specified}

Now we need $\left\langle D \psi_{i}\left|G_{\text {ret }}^{+}\right| D \psi_{i}\right\rangle$. We assume that $\psi_{i}=\psi_{n l m}$ is one of the strongly bound, compact states of small $n$ and small $l$. Such states extend only a few bohrs from the nucleus. We will actually construct $G_{\text {ret }}^{+}\left|D \psi_{i}\right\rangle$, or, more precisely, we will construct

$$
\psi_{\text {ret }}(\mathbf{q}) \equiv \int G_{\text {ret }}^{+}\left(\mathbf{q}, \mathbf{q}^{\prime} ; E\right) D\left(\mathbf{q}^{\prime}\right) \psi_{i}\left(\mathbf{q}^{\prime}\right) d \mathbf{q}^{\prime}
$$

for $\mathbf{q}$ in the same small region close to the nucleus.

To calculate $\psi_{\text {ret }}(\mathbf{q})$, the following reasoning is used. $G_{\text {dir }}^{+}\left|D \psi_{i}\right\rangle$ is a wave that propagates outward from the vicinity of the nucleus where $\left|D \psi_{i}\right\rangle$ is substantial. At $r=50 a_{0}$, these outgoing quantum waves are joined to semiclassical waves correlated with trajectories. With each trajectory that returns to the sphere $r=50 a_{0}$, there is an associated returning semiclassical wave function. At this distance the returning semiclassical wave function is similar to a cylindrical Coulomb-scattering wave function $\psi_{c, \theta_{f}}^{m}$ defined in Sec. IV D. This wave continues to propagate inward according to formulas given there. Thus a returning wave is correlated with each closed orbit, and the full returning wave $\psi_{\text {ret }}(q)$ is the sum of such returning waves.

\section{The direct part produces an outgoing wave}

With the source points $\mathbf{q}^{\prime}$ only a few bohrs from the nucleus, let us now evaluate the outgoing waves arising from $G_{\mathrm{dir}}^{+}=G_{c}^{+}$at points $\mathrm{q}$ around $50 \mathrm{bohr}$ from the nucleus. In this region the diamagnetic field can still be neglected, asymptotic formulas for radial functions can be used, and the semiclassical approximation becomes accurate.

We use the partial wave expansion for $G_{c}^{+}\left(\mathbf{q}, \mathbf{q}^{\prime} ; E\right)$, with the zero-energy radial functions, Eqs. (4.4)-(4.5), and we use the asymptotic approximation for the outgoing Hankel function (4.6) to obtain

$$
G_{\mathrm{dir}}^{+}\left(\mathbf{q}, \mathbf{q}^{\prime} ; E=0\right) \sim-i(\pi)^{1 / 2} 2^{3 / 4} \frac{e^{i(8 r)^{1 / 2}}}{r^{3 / 4}}\left(\sum_{l, m} Y_{l m}^{*}\left(\theta^{\prime}, \phi^{\prime}\right) Y_{l m}(\theta, \phi) \frac{J_{2 l+1}\left(\left(8 r^{\prime}\right)^{1 / 2}\right)}{\left(r^{\prime}\right)^{1 / 2}} e^{-i(l+3 / 4) \pi}\right) .
$$

It is convenient to regard this Green's function as a superposition of cylindrical functions $G_{\mathrm{dir}}^{(m)+}$

$$
\begin{aligned}
& G_{\mathrm{dir}}^{+}\left(\mathbf{q}, \mathbf{q}^{\prime} ; E=0\right) \equiv \sum_{m} G_{\mathrm{dir}}^{(m)+}\left(r, \theta ; r^{\prime} \theta^{\prime} \phi^{\prime}\right) e^{i m \phi}, \\
& G_{\mathrm{dir}}^{(m)+}\left(r, \theta ; r^{\prime} \theta^{\prime} \phi^{\prime}\right)=-i(\pi)^{1 / 2} 2^{3 / 4}\left(\sum_{l \geq|m|} Y_{l m}^{*}\left(\theta^{\prime}, \phi^{\prime}\right) \frac{J_{2 l+1}\left(\left(8 r^{\prime}\right)^{1 / 2}\right)}{\left(r^{\prime}\right)^{1 / 2}} Y_{l m}(\theta, \phi=0) e^{-i(l+3 / 4) \pi}\right) \frac{e^{i(8 r)^{1 / 2}}}{r^{3 / 4}} .
\end{aligned}
$$


When $G_{\mathrm{dir}}^{+}$acts upon $|D \psi\rangle$, it gives an outgoing wave

$$
\psi_{\text {out }}(\mathbf{q})=\int G_{\text {dir }}^{+}\left(\mathbf{q}, \mathbf{q}^{\prime} ; E\right) D\left(\mathbf{q}^{\prime}\right) \psi_{i}\left(\mathbf{q}^{\prime}\right) d \mathbf{q}^{\prime} \text {. }
$$

This outgoing wave can also be decomposed into cylindrical waves,

$$
\begin{aligned}
& \psi_{\mathrm{out}}(\mathbf{q})=\sum_{m} \psi_{\mathrm{out}}^{(m)}(r, \theta) e^{i m \phi}, \\
& \psi_{\mathrm{out}}^{(m)}(r, \theta)=\int G_{\mathrm{dir}}^{(m)+}\left(r, \theta ; r^{\prime} \theta^{\prime} \phi^{\prime}\right) D\left(\mathbf{q}^{\prime}\right) \psi_{i}\left(\mathbf{q}^{\prime}\right) d \mathbf{q}^{\prime}
\end{aligned}
$$

\section{The outgoing wave is joined to a semiclassical wave}

We now wish to continue this wave into the region where the diamagnetic field cannot be neglected, using the semiclassical approximation. To do this, we define a boundary radius $r_{b}$ which is large enough that the semiclassical approximation is valid, but small enough that the diamagnetic term can be neglected. (For $B \sim a$ few tesla, any distance between $30 a_{0}$ and $100 a_{0}$ is accepted; we took $r_{b}=50 a_{0}$.) We regard each cylindrical component $\Psi_{\text {out }}^{(m)}(\mathbf{q})$ as an "initial wave" on the surface $r=r_{b}$, and we propagate each of these waves outward using the semiclassical method described in Sec. III.

The value of the wave function $\Psi_{\text {out }}^{(m)}(q)$ on the initial surface is determined from Eq. (5.5b). The initial momentum can be taken to be

$$
\begin{aligned}
& p_{r}=\left[2 m_{e}\left[E+\frac{1}{r}-\frac{p_{\phi}^{2}}{2 m_{e} r^{2}}\right]\right]^{1 / 2}, \\
& p_{\theta}=0 \\
& p_{\phi}=m \hbar .
\end{aligned}
$$

Then from the boundary $r_{b}$ we propagate a field of outgoing classical trajectories. Outside $r_{b}$ the cylindrical wave functions $\Psi_{\text {out }}^{(m)}(q)$ are obtained from Eq. (3.6).

\section{The semiclassical wave returns}

The trajectories propagate outward to large distances and are turned back by the magnetic field. Later, at various times, some of them return to cross the sphere $r_{b}$. Each group of returning trajectories is identified by a central trajectory that enters the sphere with $p_{\theta}=0$. That central trajectory is called a closed orbit. (If $m=0$ the closed orbit returns exactly to the nucleus. For $m \neq 0$ it does not reach the nucleus, but still it serves to identify the group of returning trajectories.)

The closed orbits can be labeled by two indices $\left(m, k_{m}\right)$-we speak of the $k_{m}$-th closed orbit in the subspace in which $L_{z}=m \hbar$. Each such closed orbit is surrounded by a pencil of nearby rays. The pencil of rays is the family of trajectories that initially are close enough to the closed orbit that each trajectory in the family returns to cross the final sphere almost simultaneously. Trajec- tories outside this pencil miss the sphere.

Associated with this pencil of rays is a wave function, which is calculated following the rules stated in Sec. III. This wave function also bears the labels $\left(m, k_{m}\right)$ associated with the central closed orbit in the group of rays. If $S_{2}^{m k_{m}}(r, \theta)$ and $A_{2}^{m k_{m}}(r, \theta)$ are the two-dimensional characteristic function and classical-amplitude function associated with the $\left(m, k_{m}\right)$ th pencil of rays, then the associated wave function is given by

$$
\begin{aligned}
\psi^{m k_{m}}(r, \theta, \phi)= & e^{i m \phi} A_{2}^{m k_{m}}(r, \theta) \\
& \times \exp \left[i S_{2}^{m k_{m}}(r, \theta) / \hbar-\mu^{m k_{m}} \pi / 2\right] \\
& \times \frac{r_{b}}{r}\left|\frac{\sin \theta_{i}^{m k_{m}}(r, \theta)}{\sin \theta}\right|^{1 / 2} \\
& \times \psi_{\text {out }}^{(m)}\left(r_{b}, \theta_{i}^{m k_{m}}(r, \theta)\right) .
\end{aligned}
$$

The last factor is the outgoing wave function on the initial surface, evaluated at the point $\theta_{i}^{m k_{m}}(r, \theta)$, where the trajectory that goes to the point $(r, \theta)$ originates. [Initially the pencil of rays is so narrow that we can practically say that $\theta_{i}^{m k_{m}}(r, \theta) \simeq \theta_{i}^{m k_{m}}$, the initial point of the $\left(m, k_{m}\right)$ th closed orbit.]

When the $\left(m, k_{m}\right)$ th pencil of rays returns to the initial sphere, it carries with it the wave function (5.7a). We now make an approximation for the returning wave.

For this purpose let us temporarily distinguish between the initial sphere, $r=r_{i}$, and the final sphere, $r=r_{f}$ (later they will be set equal). In the vicinity of the final sphere, the returning wave in $(5.7 \mathrm{a})$ is approximately a cylindrical Coulomb wave of the type described in Sec. IV D 3, and given quantitatively by Eq. (4.23a). In this approximation, Eq. (5.7) is equal to a constant times Eq. (4.23a). This constant can be found by evaluating both formulas at the point $\left(r_{f} \theta_{f}^{m k_{m}}\right)$, at which the $\left(m, k_{m}\right)$ th closed orbit enters the final sphere. Let us simplify the notation in (5.7a) by defining

$$
\begin{aligned}
A_{2}^{m k_{m}} & =A_{2}^{m k_{m}}\left(r_{f}, \theta_{f}^{m k_{m}}\right) \\
S_{2}^{m k_{m}} & =S_{2}^{m k_{m}}\left(r_{f}, \theta_{f}^{m k_{m}}\right) \\
\psi_{\mathrm{ret}}^{m k_{m}}= & \psi^{m k_{m}}\left(r_{f}, \theta_{f}^{m k_{m}}, \phi\right) \\
= & \left.\exp (i m \phi) A_{2}^{m k_{m}} \exp i \mid S_{2}^{m k_{m}} / \hbar-\frac{\pi}{2} \mu^{m k_{m}}\right) \\
& \times \frac{r_{f}}{r_{i}}\left|\frac{\sin \theta_{i}^{m k_{m}}}{\sin \theta_{f}^{m k_{m}}}\right|^{1 / 2} \psi_{\mathrm{out}}^{(m)}\left(r_{i}, \theta_{i}^{m k_{m}}\right) .
\end{aligned}
$$

Our final approximation asserts that for $r$ close to $r_{f}$ and $\theta$ close to $\theta_{f}^{m k_{m}}$,

$$
\psi_{\mathrm{ret}}^{m k_{m}}(r, \theta, \phi) \simeq N\left(\psi_{c, \theta_{f}^{m k_{m}}}^{m}\right)_{\mathrm{inc}}(r, \theta, \phi)
$$


where $N$ is a constant, evaluated at $\left(r_{f}, \theta_{f}^{m k_{m}}\right)$ using Eq. (4.23a),

$$
\begin{aligned}
N=\psi_{\mathrm{ret}}^{m k^{m}}\left(r_{f}, \theta_{f}^{m k_{m}}, \phi\right) /\left(\psi_{c, \theta_{f}^{m}}^{m k_{m}}\right)_{\mathrm{inc}}\left(r_{f}, \theta_{f}^{m k_{m}}, \phi\right)= & A_{2}^{m k_{m}} \operatorname{expi}\left(S_{2}^{m k_{m}} / \hbar-\mu^{m k_{m}} \pi / 2\right) \psi_{\mathrm{out}}^{m}\left(r_{i}, \theta_{i}^{m k_{m}}\right) \frac{r_{f}}{r_{i}}\left|\frac{\sin \theta_{i}^{m k_{m}}}{\sin \theta_{f}^{m k_{m}}}\right|^{1 / 2} \\
& \times\left[(-1)^{m} e^{i \pi / 2} \frac{1}{\left(2^{3 / 2} \pi\right)} \frac{1}{\left(\sin \theta_{f}\right)^{1 / 2}} \frac{1}{\left(r_{f} \sin \theta_{f}\right)^{1 / 2}} e^{-i\left(8 r_{f}\right)^{1 / 2}}\right]^{-1}
\end{aligned}
$$

The same constant multiplies the partial-wave expansion (4.22c), giving the partial-wave expansion of the returning wave close to the nucleus,

$\psi_{\mathrm{ret}}^{m k^{m}}(r, \theta, \phi)=\sum_{l} a_{l m} Y_{l m}(\theta, \phi) \frac{J_{2 l+1}\left((8 r)^{1 / 2}\right)}{r^{1 / 2}}$

where

$$
\begin{aligned}
a_{l m}= & \pi^{2} 2^{3}\left(r_{f}\right)^{1 / 2} \sin \theta_{f}(-1)^{l} e^{-i \pi / 2} e^{i\left(8 r_{f}\right)^{1 / 2}} Y_{l m}^{*}\left(\theta_{f}^{m k_{m}}, 0\right) \\
& \times A_{2}^{m k_{m}} \exp \left[i\left(S_{2}^{m k_{m}} / \hbar-\frac{1}{2} \pi \mu^{m k_{m}}\right)\right]\left[\frac{r_{f}}{r_{i}}\right] \\
& \times\left|\frac{\sin \theta_{i}^{m k_{m}}}{\sin \theta_{f}^{m k_{m}}}\right|^{1 / 2} \psi_{\text {out }}^{(m)}\left(r_{i}, \theta_{i}^{m k_{m}}\right)
\end{aligned}
$$

There are two ways to test the accuracy of this approximation. (1) Compute the returning wave as a function of $r_{f}$ and $\theta_{f}$ using Eq. (5.7) and compare it to the cylindrically modified zero-energy Coulomb wave (4.23a); (2) evaluate the coefficients $a_{l m}$ at various values of the final radius $r_{f}$ and see if they are independent of $r_{f}$. In Appendix B, we shall show that by these tests the approximation is accurate to within a few percent. In addition, we can show analytically that within these approximations, $a_{l m}$ is independent of $r_{i}$ and $r_{f}$.
In the following formulas we no longer distinguish between $r_{i}$ and $r_{f}$; they are taken to be equal, and they are denoted $r_{b}$.

\section{The returning part is the sum of returning waves}

In the vicinity of the nucleus, the full returning wave $\psi_{\text {ret }}(r, \theta, \phi)$ is the sum of contributions arising from each closed orbit,

$$
\psi_{\mathrm{ret}}(r, \theta, \phi)=\sum_{m, k_{m}} \psi_{\mathrm{ret}}^{m k_{m}}(r, \theta, \phi)
$$

We now combine Eqs. (5.8), which express $\psi_{\text {ret }}^{m k_{m}}$ in terms of $\psi_{\text {out }}^{m}$, with Eq. (5.5b), which expresses $\psi_{\text {out }}^{m}$ in terms of $G_{\mathrm{dir}}^{m+}\left|D \psi_{i}\right\rangle$. We combine the resulting expression with Eq. (5.4b), which gives an explicit form for $G_{\mathrm{dir}}^{+}$.

The result is a formula for $\psi_{\text {ret }}(r, \theta, \phi)$ which applies in the small region close to the nucleus, where $\left|\psi_{\text {ret }}\right\rangle$ overlaps with $\left\langle D \psi_{i}\right|$. In that formula $\psi_{\mathrm{ret}}(r, \theta, \phi)$ is expressed in terms of a linear integral operator acting on $\left|D \psi_{i}\right\rangle$. This linear integral operator is by definition $G_{\text {ret }}^{+}\left(\mathrm{q}, \mathrm{q}^{\prime} ; E=0\right)$ for $\mathrm{q}$ and $\mathbf{q}^{\prime}$ close to the nucleus.

The formula so obtained [by combining Eqs. (5.9a), $(5.5 b)$, and $(5.4 b)]$ is

$$
\psi_{\text {ret }}(\mathbf{q})=\int G_{\text {ret }}^{+}\left(\mathbf{q}, \mathbf{q}^{\prime} ; E\right) D\left(\mathbf{q}^{\prime}\right) \psi_{i}\left(\mathbf{q}^{\prime}\right) d \mathbf{q}^{\prime}
$$

with

$$
G_{\mathrm{ret}}^{+}\left(\mathbf{q}, \mathbf{q}^{\prime} ; E\right)=\sum_{m, k_{m}} \sum_{l_{1}, l_{2}} d_{(\geq|m|)}^{m k_{l_{1} l_{2}^{m}}} Y_{l_{2} m}^{*}\left(\theta^{\prime}, \phi^{\prime}\right) \frac{J_{2 l_{2}+1}\left(\left(8 r^{\prime}\right)^{1 / 2}\right)}{\left(r^{\prime}\right)^{1 / 2}} Y_{l_{1} m}(\theta, \phi) \frac{J_{2 l_{1}+1}\left((8 r)^{1 / 2}\right)}{r^{1 / 2}}
$$

where

$$
\begin{aligned}
d_{l_{1} l_{2}}^{m k_{m}}= & (-1)^{l_{1}+l_{2}+1} 2^{15 / 4} \pi^{5 / 2} r_{b}^{-1 / 4}\left(\sin \theta_{f}^{m k_{m}} \sin \theta_{i}^{m k_{m}}\right)^{1 / 2} Y_{l_{1} m}^{*}\left(\theta_{f}^{m k_{m}}, 0\right) Y_{l_{2} m}\left(\theta_{i}^{m k_{m}}, 0\right) A_{2}^{m k_{m}} \\
& \times e^{-i(3 / 4) \pi} e^{2 i\left(8 r_{b}\right)^{1 / 2}} \exp \left[i\left(S_{2}^{m k_{m}} / \hbar-\frac{1}{2} \pi \mu^{m k_{m}}\right)\right] .
\end{aligned}
$$

At last we have an expression for $G_{\text {ret }}^{+}$that holds in the necessary region. Now we only need its matrix elements to obtain the oscillator-strength density. 


\section{Formulas for the oscillator-strength density are given}

The oscillator-strength density is given as a function of energy by a smooth background plus a sum of oscillatory contributions, each associated with a closed orbit of the system,

$$
\begin{gathered}
D f(E)=D f_{0}(E)+\sum_{m, k_{m}} C_{m k_{m}}(E) \sin \left[\int_{0}^{E} T_{m k_{m}}\left(E^{\prime}\right) d E^{\prime}\right. \\
\left.+\Delta_{m k_{m}}\right) .
\end{gathered}
$$

\section{The direct term gives the smooth background}

As stated in Sec. IV, we assume that the initial state is a hydrogenic wave function given by Eq. (4.1). The dipole operator acts upon it to produce a new linear combination of $Y_{l m}$ 's given by Eq. (4.3) [the coefficients $b_{l m}^{i}$ are given in Eq. (4.2)].

Let $D f_{0}(E)$ represent that part of the oscillatorstrength density that arises from the direct part of the Green's function,

$D f_{0}(E)=\left[-2 m_{e}\left(E-E_{i}\right) / \pi \hbar^{2}\right] \operatorname{Im}\left\langle D \psi_{i}\left|\hat{G}+{ }_{\mathrm{dir}}^{+}\right| D \psi_{i}\right\rangle$.

The direct part of $\hat{G}^{+}$is simply the outgoing zero-energy Coulomb Green's function given in Eqs. (4.9)-(4.11). From those equations, together with Eq. (4.12), one obtains

$D f_{0}(E=0)=-\frac{4 m_{e} E_{i}}{\hbar^{2}} \sum_{l^{\prime}, m}\left|b_{l^{\prime} m}^{i} I\left(n, l, l^{\prime}\right)\right|^{2}$.
As in Eq. (4.3) the sum over $l^{\prime}$ includes only $l^{\prime}=l-1$ and $l+1$. Equation (5.10), representing the smooth background to the oscillator-strength density, is the result that would be obtained in the absence of a magnetic field.

\section{Each returning wave contributes an oscillation}

Let $D f_{1}(E)$ represent the contribution to the oscillator strength that arises from the returning part of the Green's function,

$$
D f_{1}(E)=\left[-2 m_{e}\left(E-E_{i}\right) / \pi \hbar^{2}\right] \operatorname{Im}\left\langle D \psi_{i}\left|\hat{G}_{\text {ret }}^{+}\right| D \psi_{i}\right\rangle .
$$

From (5.9), (4.3), and (4.12), straightforward algebra gives

$$
\begin{aligned}
D f_{1}(E)=\sum_{m, k_{m}} \operatorname{Im}\left(-\frac{2 m_{e}\left(E-E_{i}\right)}{\pi \hbar^{2}}\right. \\
\times \sum_{l_{1}, l_{2} d_{l_{1} l_{2}}^{m k_{m}} b_{l_{1} m}^{i *} b_{l_{2} m}^{i} I\left(n, l, l_{1}\right)} \\
\left.\times I\left(n, l, l_{2}\right)\right] .
\end{aligned}
$$

We define the quantity in large parentheses to be

$$
C_{m k_{m}}(E) e^{i \Delta_{m k_{m}}(E)} \text {, }
$$

with $C_{m k_{m}}(E)$ real and positive. Using the expression for $d_{l_{1} l_{2}}^{m k_{m}}$, Eq. (5.9b), in (5.12), and collecting sums over $l_{1}$ and $l_{2}$, we obtain

$$
\begin{aligned}
C_{m k_{m}}(E) \exp \left[i \Delta_{m k_{m}}(E)\right]= & \frac{m_{e}}{\hbar^{2}}\left(E-E_{i}\right) 2^{19 / 4} \pi^{3 / 2} r_{b}^{-1 / 4}\left[\sin \theta_{i}^{m k_{m}} \sin \theta_{f}^{m k_{m}}\right]^{1 / 2} A_{2}^{m k_{m}} e^{-i(3 / 4) \pi} \\
& \times e^{2 i\left(8 r_{b}\right)^{1 / 2}} \exp \left[i\left(S_{2}^{m k_{m}} / \hbar-\frac{1}{2} \pi \mu^{m k_{m}}\right)\right] \mathcal{Y}\left(\theta_{i}^{m k_{m}}\right) \mathcal{Y}^{*}\left(\theta_{f}^{m k_{m}}\right),
\end{aligned}
$$

where

$$
\mathcal{Y}(\theta)=\sum_{l_{1}}(-1)^{l_{1}} I\left(n, l, l_{1}\right) b_{l_{1} m}^{i} Y_{l_{1} m}(\theta, 0) .
$$

It follows that the oscillator-strength density can be written in the form

$D f(E)=D f_{0}(E)+\sum_{m, k_{m}} C_{m k_{m}}(E) \sin \Delta_{m k_{m}}(E)$.

How do these quantities vary as a function of energy? All of the significant energy dependence is contained in the coefficient $d_{l_{1} l_{2}}^{m k_{m}}$, defined in Eq. (5.9b). This quantity is determined from the $k_{m}$-th closed orbit having $L_{z}=m \hbar$ that goes out from polar angle $\theta_{i}^{m k^{m}}$ and returns to polar angle $\theta_{f}^{m k_{m}}$. As the energy changes, the initial and final angles associated with the closed orbit will change. This will produce a small change in the spherical harmonics $Y_{l m}\left(\theta_{i}^{m k_{m}}, 0\right)$ and a small change in the classical amplitude $A_{2}^{m k_{m}}$. The Maslov index is a topological property of the $k_{m}$-th family of orbits, and it will not change with small changes of energy. Compared to the preceding relatively weak dependence on energy, the action $S_{2}$ along a closed orbit changes very rapidly. In Appendix $C$, it is proved that the action along a closed orbit satisfies

$$
\frac{\partial S_{2}(E)}{\partial E}=T(E)
$$

where $T(E)$ is the travel time along the closed orbit. We can therefore write the oscillator-strength density around $E=0$ as

$$
\begin{aligned}
D f(E)=D f_{0}(E)+\sum_{m, k_{m}} & C_{m k_{m}}(E) \\
\times \sin [ & \int_{0}^{E} T_{m k_{m}}(E) d E / \hbar \\
& \left.\quad+\Delta_{m k_{m}}(E=0)\right] .
\end{aligned}
$$

Furthermore, since $D f_{0}(E), C_{m k_{m}}(E)$, and $T_{m k_{m}}(E)$ are 
nearly constant over a small range of energy, we have (for $E$ close to zero),

$$
\begin{aligned}
D f(E) \simeq D f_{0}(E=0) & \\
& +\sum_{m, k_{m}} C_{m k_{m}}(E=0) \sin \left[T_{m k_{m}}(E=0) E / \hbar\right. \\
& \left.+\Delta_{m k_{m}}(E=0)\right] .
\end{aligned}
$$

We have arrived at the formula for the spectrum. Readers who have arrived with us may wish to celebrate by looking again at Fig. 3 of paper I, where the first successes of this formula are shown: the coefficients $C_{m k_{m}}$ are compared with the Fourier transform of the observed absorption spectrum.

\section{CONCLUSION: ALGORITHM FOR COMPUTING THE OSCILLATOR-STRENGTH DENSITY}

If the initial state and laser polarization are specified, then the absorption spectrum near the ionization threshold can be calculated by the following procedure.

(i) Evaluate the expansion coefficients $b_{l m}^{i}$ for $D \psi_{i}$ the product of the dipole operator $D$ and the initial state $\psi_{i}$ - according to (2.2b), (4.2), and (4.3).

(ii) Calculate the radial overlap integral $I\left(n, l, l^{\prime}\right)$ for $l^{\prime}=l \pm 1$ as in (4.12). Some of these integrals are listed in Table I.

(iii) Calculate the smooth background term in the spectrum using Eq. (5.10).

(iv) Find all the closed orbits at energy $E=0$ with period $T$ less than a desired value $T_{\max }$ in each subspace of $m$ that appears in the expansion of $D \psi_{i}$ in (4.3). For any $m$, an orbit is said to be closed if it begins radially outward on the sphere $r=r_{b}$, and ends radially inward on the same sphere. For each such closed orbit, compute the amplitude factor $A_{2}$, action $S_{2}$, Maslov index, and period $T$.

(v) From (5.13), evaluate the oscillation amplitude $C_{m k_{m}}$ and phase $\Delta_{m k_{m}}$.

(vi) The spectrum near the ionization threshold is then given by (5.16).

Obvious modifications of this procedure can be made to obtain a spectrum around any energy $E$ other than the threshold.

\section{ACKNOWLEDGMENTS}

One of us (J.B.D.) acknowledges financial support from the Joint Institute for Laboratory Astrophysics, National Bureau of Standard and University of Colorado Visiting Fellow program and he especially acknowledges the hospitality of the Institute. This work was also supported by the National Science Foundation through a grant to the College of William and Mary.

\section{APPENDIX A: A FORMULA FOR THE INTEGRAL $I\left(n, l, l^{\prime}\right)$}

Here we shall derive a general formula for the integral

$$
I\left(n, l, l^{\prime}\right)=\int_{0}^{\infty} R_{n l}(r) r^{3} \frac{J_{2 l^{\prime}+1}\left((8 r)^{1 / 2}\right)}{r^{1 / 2}} d r .
$$

Since the radial function $R_{n l}(r)$ is a product of $e^{-r / n}$ and a polynomial of order $(n-1)$,

$$
R_{n l}(r)=e^{-r / n} \sum_{k=0}^{n-1} a_{k} r^{k}
$$

the integral $I\left(n, l, l^{\prime}\right)$ is a linear combination of integrals

$$
F\left(n, k, l^{\prime}\right)=\int_{0}^{\infty} e^{-r / n} r^{k} \frac{J_{2 l^{\prime}+1}\left((8 r)^{1 / 2}\right)}{r^{1 / 2}} d r
$$

for $k$ going from 3 to $(n+2)$,

$$
I\left(n, l, l^{\prime}\right)=\sum_{k=3}^{N+2} a_{k-3} F\left(n, k, l^{\prime}\right) \text {. }
$$

To evaluate $F\left(n, k, l^{\prime}\right)$, we first make a change of variable $x=(8 r)^{1 / 2}$ in the integral; then

$F\left(n, k, l^{\prime}\right)=\frac{1}{4 \times 8^{k-1 / 2}} \int_{0}^{\infty} e^{-x^{2} / 8 n} x^{2 k} J_{2 l^{\prime}+1}(x) d x$.

Now we use formula (11.4.28) of Ref. 7,

$$
\begin{aligned}
& \int_{0}^{\infty} e^{-a^{2} t^{2} t^{\mu-1} J_{v}(b t) d t} \\
&=\frac{\Gamma\left(\frac{1}{2} v+\frac{1}{2} \mu\right)\left(\frac{1}{2} b / a\right)^{v}}{2 a^{\mu} \Gamma(v+1)} M\left(\frac{1}{2} v+\frac{1}{2} \mu, v+1,-b^{2} / 4 a^{2}\right) \\
& \quad\left[\operatorname{Re}(\mu+v)>0, \quad \operatorname{Re} a^{2}>0\right],
\end{aligned}
$$

where the notation $M(a, b, z)$ stands for the confluent hypergeometric function, which has a Taylor expansion

$M(a, b, z)=1+\frac{a z}{b}+\frac{(a)_{2} z^{2}}{(b)_{2} 2 !}+\cdots+\frac{(a)_{n} z^{n}}{(b)_{n} n !}+\cdots$,

with the Pochhammer symbol

$(a)_{n}=a(a+1)(a+2) \cdots(a+n-1), \quad$ with $(a)_{0}=1$.

The result for $F\left(n, k, l^{\prime}\right)$ is

$$
\begin{aligned}
F\left(n, k, l^{\prime}\right)= & 2^{l^{\prime}+1 / 2} n^{k+l^{\prime}+1} \frac{\Gamma\left(k+l^{\prime}+1\right)}{\Gamma\left(2 l^{\prime}+2\right)} \\
& \times M\left(k+l^{\prime}+1,2 l^{\prime}+2,-2 n\right) .
\end{aligned}
$$

\section{APPENDIX B: TESTS OF THE APPROXIMATION FOR RETURNING WAVES}

Equation (5.7e) asserts that the semiclassical returning wave, described by Eqs. (5.7a)-(5.7d) is approximately equal to a constant times the incoming part of a cylindrically modified zero-energy Coulomb wave, which is given by Eq. (4.23a). Here we display results of tests of the accuracy of this approximation. 


\section{A. Direct comparison}

We have numerically computed the returning semiclassical wave associated with the family of trajectories surrounding closed orbit number 2 (the orbit shown in Fig. 1). To compute this returning wave we took the initial state to be $2 s$. We have also numerically computed the modified Coulomb wave (4.23a). The "constant" $N$ in Eq. (5.7e) was evaluated by comparing the two waves at the point $r_{f}=50 a_{a}, \theta_{f}=53.8315^{\circ}$.

The two wave functions are compared on two slices through configuration space: on the circle $r=50 a_{0}$ with angle varying, and on the line $\theta=\theta_{f}$ with radius varying. Results are shown in Fig. 4. The open and closed circles are the semiclassical returning wave, while the solid lines
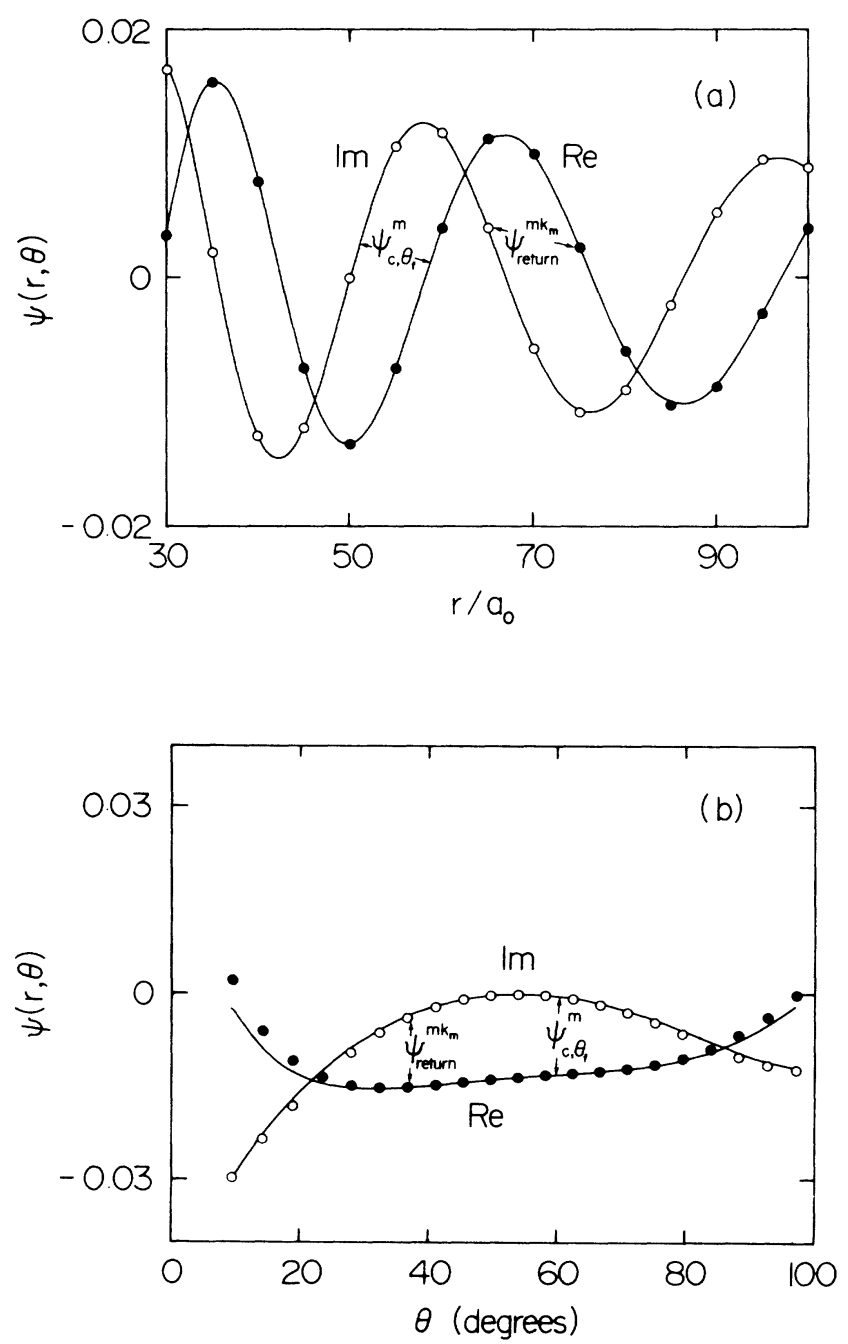

FIG. 4. Test of the returning-wave approximation. The closed and open circles are, respectively, the real and imaginary parts of a returning wave as calculated by the semiclassical method. The solid lines represent the real and imaginary parts of $\psi_{c, \theta_{f}}^{m=0}(r, \theta)$. These are compared (a) as a function of $r$ at the fixed angle $\theta=\theta_{f}=53.8315^{\circ}$ and (b) as a function of $\theta$ at the fixed radius $r=50 a_{0}$. The complex constant was chosen so that the two wave functions would exactly match at the point $r=50 a_{0}, \theta=\theta_{f}$. are the modified Coulomb wave (4.23a). The agreement between the two is very good.

\section{B. Dependence of $a_{l m}$ on boundary radius}

If Eq. (5.7e) were exact rather than an approximation, then the coefficients $a_{l m}$ defined in Eqs. (5.8a) and (5.8b) would be independent of the boundary radius $r_{f}$. We varied the boundary radius between 30 and $100 a_{0}$, and obtained the results shown in Table II. The phase of $a_{10}$ changes negligibly with $r_{f}$, and the amplitude changes by about $3 \%$. This should be a good measure of the accuracy of the returning-wave approximation for this group of trajectories, and it makes us believe that our amplitudes $C_{m k_{m}}$ are accurate to within a few percent.

\section{APPENDIX C: ACTION AND TIME THEOREM}

Consider a trajectory with energy $E_{1}$ going from $\mathrm{q}^{\prime}$ to $\mathbf{q}^{\prime \prime}$. When the energy is changed from $E_{1}$ to energy $E_{2}$ which is close to $E_{1}$, the trajectory going from $\mathbf{q}^{\prime}$ to $\mathbf{q}^{\prime \prime}$ will follow a different but nearby path [see Fig. 6(c) of Ref. 1]. How are the actions along the two paths related?

Theorem. We have

$$
\left(\frac{\partial S}{\partial E}\right)_{q^{\prime}, q^{\prime \prime}}=T(E),
$$

where $T(E)$ is the time needed to go from $\mathrm{q}^{\prime}$ to $\mathrm{q}^{\prime \prime}$.

Proof. The trajectory going from $\mathrm{q}^{\prime}$ to $\mathrm{q}^{\prime \prime}$ at energy $E$ can be written as

$$
\mathbf{q}=\mathbf{q}\left(t, E, \mathbf{q}^{\prime}, \mathbf{q}^{\prime \prime}\right), \quad \mathbf{p}=\mathbf{p}\left(t, E, \mathbf{q}^{\prime}, \mathbf{q}^{\prime \prime}\right) \text {. }
$$

The action along the trajectory is defined as

$S\left(\mathbf{q}^{\prime}, \mathbf{q}^{\prime \prime}, E\right)=\int_{t^{\prime}}^{t^{\prime \prime}} \mathbf{p}\left(t, E, \mathbf{q}^{\prime}, \mathbf{q}^{\prime \prime}\right) \frac{\partial \mathbf{q}\left(t, E, \mathbf{q}^{\prime}, \mathbf{q}^{\prime \prime}\right)}{\partial t} d t$

where, in (C3), $t^{\prime}$ and $t^{\prime \prime}$ are the times that the particle arrives at $\mathbf{q}^{\prime}$ and $\mathbf{q}^{\prime \prime}$. Taking derivatives of (C3),

$$
\frac{\partial S}{\partial E}=\int_{t^{\prime}}^{t^{\prime \prime}}\left[\frac{\partial \mathbf{p}}{\partial E} \cdot \frac{\partial \mathbf{q}}{\partial t} d t+\mathbf{p} \cdot \frac{\partial^{2} \mathbf{q}}{\partial E \partial t} d t\right)+\left.\mathbf{p} \cdot \frac{\partial \mathbf{q}}{\partial t} \frac{\partial t}{\partial E}\right|_{t^{\prime}} ^{t^{\prime \prime}}
$$

a partial integration of the second term gives us

TABLE II. Expansion coefficient $a_{10}$.

\begin{tabular}{rcc}
\hline$r_{f}\left(a_{0}\right)$ & Amplitude of $a_{10}$ & Phase of $a_{10}$ \\
\hline 30.00 & 1.723 & 344.794 \\
40.00 & 1.734 & 344.795 \\
50.00 & 1.744 & 344.795 \\
60.00 & 1.753 & 344.796 \\
70.00 & 1.761 & 344.796 \\
80.00 & 1.769 & 344.796 \\
90.00 & 1.776 & 344.796 \\
100.00 & 1.783 & 344.797 \\
\hline \hline
\end{tabular}




$$
\begin{aligned}
\frac{\partial S}{\partial E}= & \int_{t^{\prime}}^{t^{\prime \prime}}\left(\frac{\partial \mathbf{p}}{\partial E} \cdot \frac{\partial \mathbf{q}}{\partial t}-\frac{\partial \mathbf{q}}{\partial E} \cdot \frac{\partial \mathbf{p}}{\partial t}\right) d t+\left.\mathbf{p} \cdot \frac{\partial \mathbf{q}}{\partial E}\right|_{t^{\prime}} ^{t^{\prime \prime}} \\
& +\left.\mathbf{p} \cdot \frac{\partial \mathbf{q}}{\partial t} \frac{\partial t}{\partial E}\right|_{t^{\prime}} ^{t^{\prime \prime}}
\end{aligned}
$$

If the two end points $q^{\prime}$ and $q^{\prime \prime}$ are fixed,

$$
\mathbf{q}\left(t^{\prime}, E, \mathbf{q}^{\prime}, \mathbf{q}^{\prime \prime}\right)=\mathbf{q}^{\prime}, \quad \mathbf{q}\left(t^{\prime \prime}, E, \mathbf{q}^{\prime}, \mathbf{q}^{\prime \prime}\right)=\mathbf{q}^{\prime \prime} \text {, }
$$

then at the end points

$$
\left(\frac{\partial \mathbf{q}}{\partial E}+\frac{\partial \mathbf{q}}{\partial t} \frac{\partial t}{\partial E}\right)_{\mathbf{q}^{\prime}, \mathbf{q}^{\prime \prime}}=\mathbf{0}
$$

Then using Hamilton's equation in (C5),

$$
\begin{aligned}
\left(\frac{\partial S}{\partial E}\right)_{\mathbf{q}^{\prime}, \mathrm{q}^{\prime \prime}} & =\int_{t^{\prime}}^{t^{\prime \prime}}\left(\frac{\partial \mathrm{p}}{\partial E} \cdot \frac{\partial H}{\partial \mathrm{p}}+\frac{\partial H}{\partial \mathbf{q}} \cdot \frac{\partial \mathrm{q}}{\partial E}\right) d t \\
& =\int_{t^{\prime}}^{t^{\prime \prime}} \frac{\partial H}{\partial E} d t=\int_{t^{\prime}}^{t^{\prime \prime}} d t=T(E)
\end{aligned}
$$

(QED).

By the same method, Eq. (C1) can be shown to apply also to periodic trajectories. In the case of a periodic trajectory, as the energy changes, the entire periodic orbit may shift, such that the starting and ending points change with energy. Nevertheless, the theorem still holds.

In a similar way, one can show that if the Hamiltonian contains a parameter $\lambda$, then the action integral on an orbit varies as

$$
\left(\frac{\partial S}{\partial \lambda}\right)_{E, q^{\prime}, q^{\prime \prime}}=-\int \frac{\partial H}{\partial \lambda} d t .
$$

In particular, in the present case

$$
\frac{\partial S}{\partial B}=-\frac{B}{4 m_{e}}\left(\frac{e}{c}\right)^{2} \int \rho^{2} d t
$$

\section{APPENDIX D: PRACTICAL FORMULA FOR EVALUATING SEMICLASSICAL WAVE AMPLITUDE}

When computing the semiclassical amplitude $A$ of the returning wave according to (3.9) and (5.7a), the derivatives of $r$ and $\theta$ with respect to the initial angle $\theta_{0}$ are evaluated at a fixed time,

$$
\left(\frac{\partial r}{\partial \theta_{0}}\right)_{t}, \quad\left[\frac{\partial \theta}{\partial \theta_{0}}\right)_{t} .
$$

However, because usually we do not use a fixed time step in our numerical integration, we do not have the data for neighboring trajectories at the same time. In our case we launch a family of trajectories at $t=0$ from the initial circle, and each of these trajectories is propagated with a varied time step size according to the location of the electron. Trajectories are stopped on the final circle $r_{f}=$ const. Trajectories in this family do not arrive at the final circle at the same time. Let us express the desired quantities in terms of other quantities which are readily obtained from the computation.

From running the trajectories, we could, in principle, obtain two functions,

$$
\begin{aligned}
& r=r\left(t, \theta_{0}\right), \\
& \theta=\theta\left(t, \theta_{0}\right) .
\end{aligned}
$$

We have from the first one,

$$
d r=\left(\frac{\partial r}{\partial \theta_{0}}\right)_{t} d \theta_{0}+\left(\frac{\partial r}{\partial t}\right)_{\theta_{0}} d t .
$$

On the final circle $r$ is a constant, so that we obtain

$$
\left(\frac{\partial r}{\partial \theta_{0}}\right)_{t}=-\left(\frac{\partial r}{\partial t}\right)_{\theta_{0}}\left(\frac{\partial t}{\partial \theta_{0}}\right)_{r} .
$$

The quantities on the right-hand side of this equation are naturally evaluated by the program: the first factor is the radial velocity at the final surface, and the second factor is the final-time difference between adjacent trajectories divided by the initial-angle difference.

Similarly, from (D1b), we have

$$
d \theta=\left(\frac{\partial \theta}{\partial t}\right)_{\theta_{0}} d t+\left(\frac{\partial \theta}{\partial \theta_{0}}\right)_{t} d \theta_{0},
$$

which, on the final circle, is

$$
\left(\frac{\partial \theta}{\partial \theta_{0}}\right)_{t}=\left(\frac{\partial \theta}{\partial \theta_{0}}\right)_{r}-\left(\frac{\partial \theta}{\partial t}\right)_{\theta_{0}}\left(\frac{\partial t}{\partial \theta_{0}}\right)_{r} .
$$

Equations (D3) and (D5) are used in our calculation of $\mathrm{A}_{2}^{m k_{m}}$.
*Permanent address.

${ }^{1}$ M. L. Du and J. B. Delos, Phys. Rev. A 38, 1896 (1988).

${ }^{2}$ L. I. Schiff, Quantum-Mechanics, 2nd ed. (McGraw-Hill, New York, 1955).

${ }^{3}$ R. G. Newton, Scattering Theory of Waves and Particles, 2nd ed. (Springer-Verlag, Berlin, 1982).

${ }^{4}$ D. Ter Haar, Problems in Quantum Mechanics, 3rd ed. (Pion, London, 1975).

5(a) V. P. Maslov and M. V. Fedoriuk, Semi-Classical Approximation in Quantum Mechanics (Reidel, Boston, 1981). (b) J. B. Delos, Adv. Chem. Phys. 65, 161 (1986); S. K. Knudson, J. B. Delos, and B. Bloom, J. Chem. Phys. 83, 5703 (1985); S. K. Knudson, J. B. Delos, and D. W. Noid, ibid. 84, 6886 (1986); J. B. Delos, ibid. 86, 425 (1987). (c) M. C. Gutzwiller, J.
Math. Phys. 8, 1979 (1967). See also J. Math. Phys. 11, 1791 (1970); 12, 343 (1971).

${ }^{6} \mathrm{H}$. A. Bethe and E. E. Salpeter, Quantum Mechanics of Oneand Two-Electron Atoms (Plenum, New York, 1977).

${ }^{7}$ Handbook of Mathematical Functions, Natl. Bur. Stand. Appl. Math. Ser. No. 55, edited by M. Abramowitz and I. Stegun (U.S. GPO, Washington, D.C., 1965).

${ }^{8}$ L. S. Rodberg and R. M. Thaler, Introduction to the Quantum Theory of Scattering (Academic, New York, 1967); or P. M. Morse and H. Feshbach, Methods of Theoretical Physics (McGraw-Hill, New York, 1953).

${ }^{9}$ J. D. Jackson, Classical Electrodynamics (Wiley, New York, 1975). 